\title{
Contribuciones geológicas al modelo conceptual geotérmico en la región de los volcanes Chiles - Cerro Negro (Colombia-Ecuador)
}

\author{
Yohanna K. García ${ }^{1}$;ohn J. Sánchez ${ }^{2 *}$
}

DOI: http://dx.doi.org/10.18273/revbol.v41n1-2019008 @ (c)

Forma de citar: García, Y.K., y Sánchez, J.J. (2019). Contribuciones geológicas al modelo conceptual geotérmico en la región de los volcanes Chiles - Cerro Negro (Colombia-Ecuador). Boletín de Geología, 41(1), 151-171. DOI: 10.18273/revbol.v41n1-2019008

\begin{abstract}
RESUMEN
Se refina el modelo conceptual geotérmico del área del Complejo Volcánico Chiles - Cerro Negro (CVCCN), mediante la inclusión de detalles litológicos, geotécnicos y del sistema hidrológico. Se visitaron 21 sitios en su mayoría ubicados dentro del área de interés del Proyecto Geotérmico Binacional Tufiño Chiles - Cerro Negro. La petrografía y clasificación geoquímica se realizaron para cuatro muestras utilizando los diagramas $\mathrm{K}_{2} \mathrm{O}$ vs $\mathrm{SiO}_{2}, \mathrm{SiO}_{2}-\mathrm{FeO}_{\mathrm{t}} / \mathrm{MgO}, \mathrm{AFM}$ y $\mathrm{Na}_{2} \mathrm{O}+\mathrm{K}_{2} \mathrm{O} v \mathrm{~s} \mathrm{SiO}_{2}$. La clasificación geotécnica de cinco sitios siguió el procedimiento del sistema de clasificación de macizos rocosos (RMR - Rock Mass Rating). Se encontró que la litología varía entre rocas duras (lavas andesíticas y dacíticas) y depósitos de naturaleza variable incluyendo derrubios, till, lahares y flujos piroclásticos. Los parámetros geotécnicos de los macizos rocosos presentan los siguientes rangos y valores medios: $90 \leq \% R Q D \leq 96 ; 52 \leq R M R \leq 77 ; 8,89 \leq \sigma_{c m} \leq 35,67 ; \mathrm{y}-1,13 \leq \sigma_{t m} \leq-0,11$; $R Q D_{\text {mediana }}=92 \% ; R M R_{\text {mediana }}=69 \% ; \sigma_{\text {cm-mediana }}=22,87 \mathrm{MPa} ; \mathrm{y} \sigma_{\text {tm-mediana }}=-0,494 \mathrm{MPa}$. Se estima que la porosidad y la permeabilidad en el área de estudio son moderadas a altas. Se concluye que la variabilidad litológica y estructural influencian la permeabilidad regional y local y que en general se favorece la circulación de fluidos acuosos entre las áreas de recarga y descarga del sistema geotérmico.
\end{abstract}

Palabras clave: Complejo Volcánico Chiles-Cerro Negro; sistema geotérmico; modelo geotérmico conceptual.

\section{Geological contributions to the conceptual geothermal model in the region of the volcanoes Chiles - Cerro Negro (Colombia-Ecuador)}

\begin{abstract}
A preliminary conceptual geothermal model for the Chiles-Cerro Negro Volcanic Complex (CCNVC) is refined through the addition of details related to lithology, geotechnical properties, and hydrology of the system. A total of 21 sites were visited, mostly located within the area of the Tufiño-Chiles-Cerro Negro Binational Geothermal Project. The petrography and geochemical classification were obtained for four samples using the $\mathrm{K}_{2} \mathrm{O}_{v s} \mathrm{SiO}_{2}$, $\mathrm{SiO}_{2}-\mathrm{FeO}_{\mathrm{t}} / \mathrm{MgO}, \mathrm{AFM}$ and $\mathrm{Na}_{2} \mathrm{O}+\mathrm{K}_{2} \mathrm{O}$ vs $\mathrm{SiO}_{2}$ diagrams. The geotechnical classification of five sites followed the Rock Mass Rating-RMR system. Lithology varies between hard rocks (andesite and dacite lavas) and unconsolidated deposits of variable nature, including talus, till, lahars and pyroclastic flows. The geotechnical parameters of rock masses vary in the following ranges and have median values: $90 \leq \% R Q D \leq 96 ; 52 \leq R M R$ $\leq 77 ; 8.89 \leq \sigma_{c m} \leq 35.67$; and $-1.13 \leq \sigma_{t m} \leq-0.11 ; R Q D_{\text {median }}=92 \% ; R M R_{\text {median }}=69 \% ; \sigma_{c m \text {-median }}=22.87 \mathrm{MPa}$; and $\sigma_{\text {tm-median }}=-0.494 \mathrm{MPa}$. The porosity and permeability of the study area exhibit moderate to high values. It is concluded that the varying lithology and structures influence the regional and local permeability, which favors circulation of aqueous fluids between the recharge and discharge areas of the geothermal system.
\end{abstract}

Keywords: Chiles-Cerro Negro Volcanic Complex; geothermal system; conceptual geothermal model.

${ }^{1}$ Departamento de Geociencias, Facultad de Ciencias, Universidad Nacional de Colombia, Bogotá, Colombia. ykgarciaz@unal.edu.co

${ }^{2}$ Departamento de Geociencias y Medio Ambiente, Facultad de Minas, Universidad Nacional de Colombia, Medellín, Colombia. (*) jjsanchezag@unal.edu.co 


\section{INTRODUCCIÓN}

El Complejo Volcánico Chiles-Cerro Negro (CVCCN) se ubica cerca al Municipio de Cumbal (Departamento de Nariño), en la frontera con Ecuador a $80 \mathrm{~km}$ al SW de San Juan de Pasto en las coordenadas $0,82^{\circ} \mathrm{N}$ y $77,93^{\circ} \mathrm{W}$ (volcán Chiles, VC) y $0,82^{\circ} \mathrm{N}-77,95^{\circ} \mathrm{W}$ (Volcán Cerro Negro, VCN) (FIGURA 1). El sistema geotérmico Tufiño-Chiles-Cerro Negro, que incluye el CVCCN, es compartido con el vecino país Ecuador y reviste especial interés, entre otros aspectos, por el promisorio potencial de energía eléctrica en una planta planeada con capacidad instalada en exceso de 130 MW (Mejía et al., 2014), que haría parte de colaboración entre las dos naciones en el marco del Proyecto Geotérmico Binacional Tufiño-Chiles-Cerro Negro. En este trabajo se presentan resultados de: 1) exploración geológica superficial en el CVCCN para estudiar unidades litológicas; 2) análisis de estructuras geológicas, y 3) reconocimiento de manifestaciones hidrotermales y manantiales, con el objetivo de contribuir al refinamiento del modelo geotérmico conceptual de este sistema.
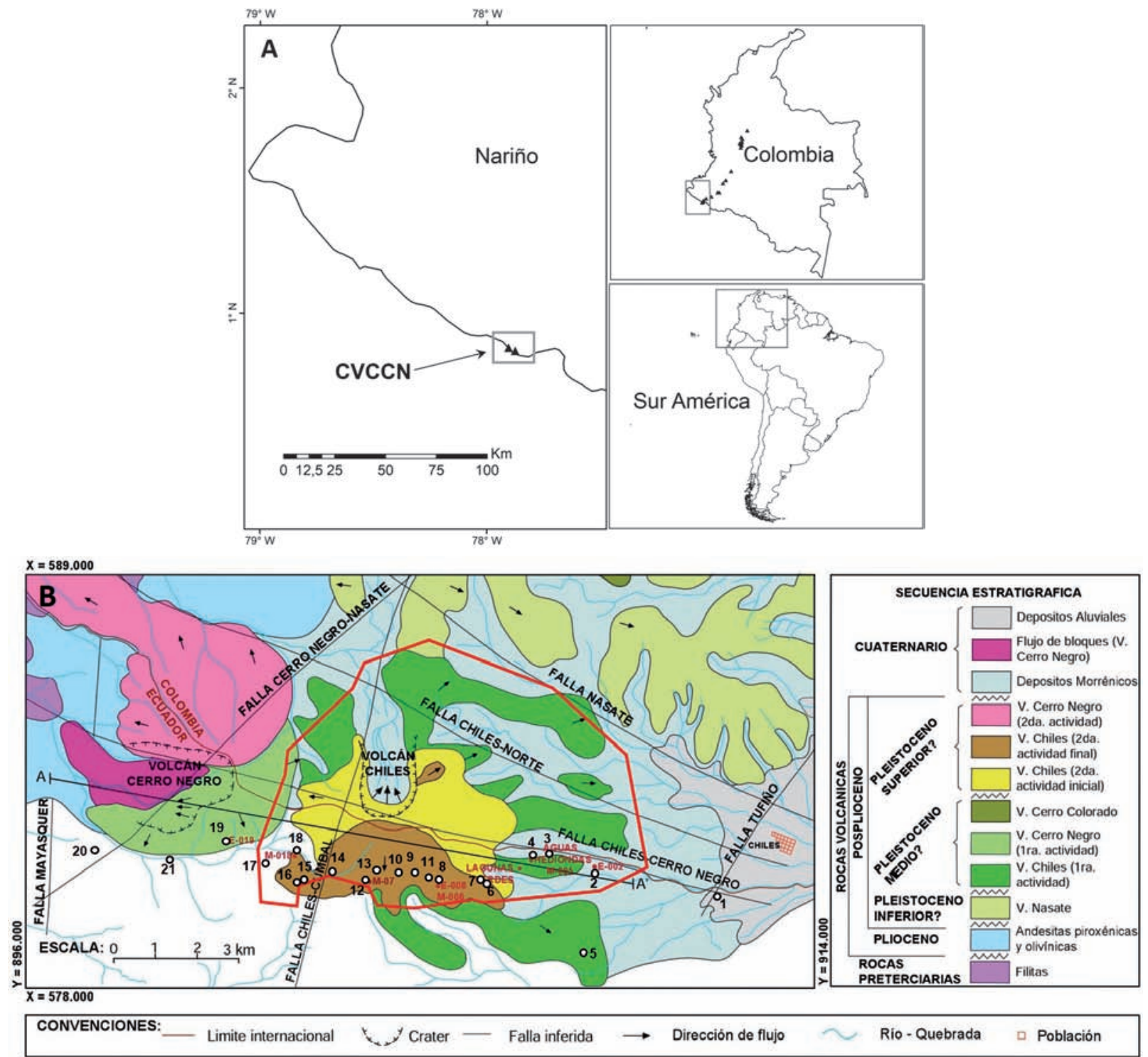

FIGURA 1. A. Mapa de localización con la ubicación del área de estudio (CVCCN). Triángulos negros: volcanes activos. B. Mapa geológico simplificado (modificado de Perdomo et al., 1986) de la región de los volcanes VC y VCN. Círculos negros numerados: Estaciones de Campo (García, 2016). Puntos rojos: Ubicación de manantiales (E-002, E-008, E019) y fuentes termales (Aguas Hediondas y Lagunas Verdes) y sitios de muestras con análisis petrográfico y químico (M-005, M-006, M-007 y M-008). Polígono rojo: Área de interés del Proyecto Geotérmico Binacional Tufiño Chiles Cerro Negro. Línea A-A': Dirección y extensión del perfil geológico mostrado en la FIGURA 8. 
La energía geotérmica es el calor que proviene del interior terrestre e impulsa fenómenos geológicos a escala global. En la actualidad, sin embargo, el término "energía geotérmica” se usa para referirse a aquella parte del calor terrestre que puede ser recuperada y explotada. Los recursos del calor geotérmico no están distribuidos uniformemente en el planeta, ya que son característicos de regiones flujo de calor elevado y circulación de fluidos, típicamente a lo largo de límites de placas litosféricas. Así es entendible que, por ejemplo, los mayores productores de electricidad derivada de energía geotérmica están en regiones de volcanismo activo. Un sistema geotérmico incluye tres rasgos principales: la fuente de calor, el reservorio y el fluido, que es el agente que transporta el calor. La fuente de calor puede ser una intrusión magmática superficial $(5 \mathrm{~km}-10 \mathrm{~km})$ con temperaturas periféricas de $600^{\circ} \mathrm{C}$ o puede ser una región con flujo de calor proveniente del gradiente geotérmico, que aumenta con la profundidad. El reservorio es un volumen de rocas permeables desde las cuales los fluidos en circulación extraen el calor y que en muchos casos está relacionado con un área superficial de recarga que reemplaza parcial o totalmente la descarga natural del sistema que ocurre a través de manantiales o fuentes termales. El fluido geotérmico es el agua, en la mayoría de los casos agua meteórica, en fase líquida o vapor que con frecuencia contiene compuestos disueltos (Dickson y Fanelli, 1995).

Los sistemas geotérmicos se han clasificado en cinco categorías de acuerdo a criterios geológicos, geofísicos, hidrológicos, e ingenieriles: 1) Sistemas ígneos jóvenes asociados con volcanismo cuaternario e intrusiones; 2) sistemas tectónicos en los cuales hay flujo de calor elevado sin actividad ígnea; 3) sistemas geopresurizados encontrados principalmente en cuencas sedimentarias; 4) sistemas de roca seca y caliente relacionados a calor almacenado en rocas impermeables de baja porosidad, y 5) sistemas de aprovechamiento de magma que involucran perforación en cuerpos magmáticos superficiales (Goff y Janik, 2000). Un aspecto clave en la exploración, desarrollo, y utilización de cualquier tipo de sistema geotérmico es la clara definición y entendimiento de su naturaleza y características, lo cual se logra óptimamente a través de la construcción de un modelo conceptual para el sistema de interés. Un modelo conceptual geotérmico es una idea descriptiva o cualitativa que incorpora los rasgos esenciales del sistema (FIGURA 2). Los modelos conceptuales geotérmicos se construyen con base en el análisis de información geológica y geofísica, datos de temperaturas y presiones, información sobre las propiedades del reservorio e información sobre la dinámica y química de los fluidos (Axelsson, 2013).

Actualmente en Colombia se desarrollan investigaciones con diferentes estados de avance en cinco áreas estratégicas con sistemas geotérmicos: 1) Volcán Nevado del Ruiz, 2) Sistema geotérmico Tufiño-Chiles-Cerro Negro; 3) Área geotérmica de Paipa; 4) Volcán Azufral, y 5) Área geotérmica San Diego (Alfaro, 2015).

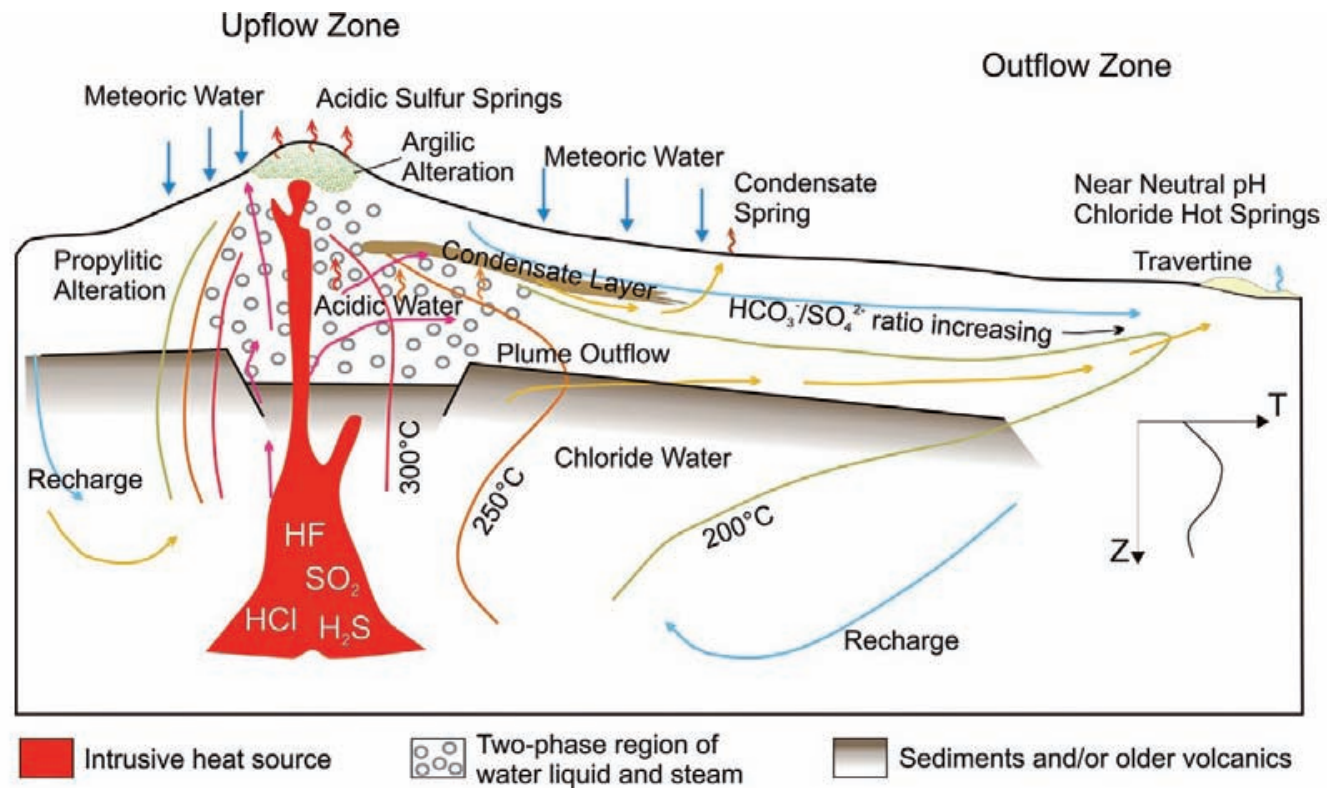

FIGURA 2. Esquema de un sistema geotérmico idealizado relacionado a un campo activo típico de arco volcánico. Tomado de Moeck (2014). 


\section{TRABAJOS CONOCIMIENTO ACTUAL DE LA GEOLOGÍA Y GEOTERMIA DEL CVCCN}

Regionalmente, la geología del CVCCN incluye rocas de los grupos de edad cretácica Dagua y Diabásico (Nelson, 1962) en la base y productos de efusiones y explosiones de varios volcanes al techo, con edad aproximada Pleistoceno Inferior para productos del VC (Pinilla et al., 2008). Con base en dataciones de $\sim 194,000$ años para las lavas más antiguas del volcán Cumbal (Gorman, 1997), argumenta que debido a la avanzada incisión del VC y su perfil de meteorización (en relación al volcán Cumbal), la base del VC debe ser más antigua que la del volcán Cumbal. En particular, el VC se encuentra en la intersección de la Falla Chiles-Cerro Negro con la Falla Chiles-Cumbal. Los afloramientos de la zona fueron caracterizados como pequeños, poco continuos y con alto grado de meteorización (Velásquez y Parra, 2002). La TABLA 1 resume los rasgos principales de ambas unidades.

TABLA 1. Resumen de rasgos principales de la geología regional del CVCCN. Modificado de Velásquez y Parra (2002).

\begin{tabular}{|c|c|}
\hline \multicolumn{2}{|r|}{ Unidades basales } \\
\hline $\begin{array}{l}\text { Grupo Dagua } \\
\text { (Nelson, 1962) }\end{array}$ & $\begin{array}{l}\text { Dos unidades: (1) Arcillolitas y areniscas de colores negros a grises, con ocasionales estratos } \\
\text { gruesos de conglomerados; (2) Limolitas silíceas de colores grises y verdes asociadas a tobas } \\
\text { volcánicas básicas y chert. }\end{array}$ \\
\hline $\begin{array}{l}\text { Grupo Diabásico } \\
\text { (Nelson, 1962) }\end{array}$ & $\begin{array}{l}\text { Basaltos y diabasas de color verde, masivas, solamente en las últimas se distinguen algunos } \\
\text { cristales milimétricos de piroxeno. }\end{array}$ \\
\hline \multicolumn{2}{|r|}{ Episodios efusivos y piroclásticos } \\
\hline Andesitas del Cumbal moderno & $\begin{array}{l}\text { Lavas andesiticas de textura porfirítica con plagioclasas, piroxenos y fragmentos de rocas } \\
\text { andesíticas con olivino. }\end{array}$ \\
\hline Depósito de avalancha (ं?) de escombros & Depósito de colapso del edificio volcánico, bloques y cantos en matriz arenosa. \\
\hline Andesitas del VC & Lavas andesíticas masivas compuestas principalmente por plagioclasas y piroxenos. \\
\hline Andesitas del VCN & $\begin{array}{l}\text { Depósitos piroclásticos de obsidiana, pómez y ceniza. } \\
\text { Lavas andesíticas con textura cordada y en bloques. }\end{array}$ \\
\hline
\end{tabular}

Los volcanes del CVCCN han presentado cada uno dos etapas de actividad similares, separadas por discordancias menores de acción glaciar. La primera etapa, incluyó la emisión de lavas predominantemente andesíticas piroxénicas y en la segunda se emplazaron lavas con claras estructuras de flujo y de composición dacítica. De acuerdo al estado de conservación de los edificios volcánicos y de sus productos se ha sugerido que el volcanismo del sistema Chiles-Cerro Negro inició en el Plioceno y ha continuado hasta el presente, alternando con algunos episodios de calma (Perdomo et al., 1986). La actividad predominantemente efusiva del VC ha sido caracterizada como una secuencia de seis episodios (Cortés y Calvache, 1996), del más antiguo al más reciente: 1) Cordones de lava andesítica con grado bajo-medio de alteración, 2) Cordones de lavas dacíticas, 3) Franja de lava alargada en dirección $\mathrm{S} 78^{\circ} \mathrm{E}$ de composición dacítica, 4) Lavas cordadas con dirección $\mathrm{S} 72^{\circ} \mathrm{E}$ de composición dacítica, 5) Lavas andesíticas con apariencia columnar alargadas en dirección $\mathrm{N} 50^{\circ} \mathrm{E}$, y 6 ) Flujos cortos de lavas dacíticas y andesíticas. De otra parte, el edificio volcánico del VCN es una secuencia de flujos de lava y flujos piroclásticos emplazados en 5 episodios (Cortés y Calvache, 1996), que del más antiguo al más reciente son: 1) lavas de composición andesítica, 2) flujo de lava en bloques de composición dacítica, 3) depósitos de flujo piroclástico, con clastos de composición dacítica, 4) flujo de lava dacítica en bloques, 5) lavas cordadas de composición dacítica. Algunos depósitos de caída son observables en los alrededores de ambos volcanes, pero la fuente correspondería al volcán Azufral o un volcán ecuatoriano. Aunque se evidencian episodios de actividad explosiva y el CVCCN está compuesto por dos volcanes ya colapsados (INGEOMINAS, 1999), estos se clasifican como estratovolcanes activos con dominio efusivo y en estado de reposo.

En lo referente a manifestaciones superficiales de actividad geotérmica, Perdomo et al. (1986) atribuyeron la alteración hidrotermal observada, a fluidos de altas temperaturas con evidencia de silicificación y argilización evidenciados por caolín y otras arcillas; dada la cercanía de los centros volcánicos y similitud en su evolución, se propone una cámara magmática común para el VC y el VCN. Lesmes et al. (2004) identifican dos fuentes termales importantes en el $\mathrm{VC}$, estas son: Aguas Hediondas (AH) y Baños Chiles (BC). Algunas características fisicoquímicas determinadas por estos autores son: $\mathrm{T}_{\mathrm{AH}}^{\circ}=46^{\circ} \mathrm{C}, \mathrm{T}^{\circ}{ }_{\mathrm{BC}}=42^{\circ} \mathrm{C} ; \mathrm{pH}_{\mathrm{AH}}=4,9, \mathrm{pH}_{\mathrm{BC}}$ $=5,3$. También encontraron diferencias en conductividad eléctrica $(\sigma, \mathrm{mS} / \mathrm{cm})$ de: $\sigma_{\mathrm{AH}}=1,80, \sigma_{\mathrm{BC}}=1,50$ por lo que se infiere mayor concentración de solidos disueltos en el 
caso de AH. El nivel de radiación gama (DRG) ) $(\mu R / h)$ en ambas fuentes termales es de 1,0. Además, los autores encontraron que las rocas del VC presentan alteración hidrotermal por acción del vapor de agua y de los gases ácidos sulfatados.

En años recientes, las empresas ISAGEN y CELEC EP participaron conjuntamente en el denominado Proyecto Geotérmico Binacional Tufiño-Chiles-Cerro Negro, firmado por los gobiernos de Colombia y Ecuador. El proyecto, en etapa de prefactibilidad (ISAGEN, 2012), ha financiado actividades que incluyen estudios geológicos, geoquímicos, hidrogeológicos y geofísicos, así como diseños de pozos de exploración, infraestructura y estudios de impacto ambiental. El área en la cual se ha desarrollado el proyecto se extiende a lo largo $490 \mathrm{~km}^{2}$, y se espera un potencial de $138 \mathrm{MW}$ (Mejía et al., 2014).

\section{METODOLOGÍA}

\section{Levantamiento geológico}

El trabajo se desarrolló teniendo en cuenta la guía metodológica para el reconocimiento geológico en campos geotérmicos (INGEOMINAS, 2009a), que se resume a continuación. Debido a la finalidad y alcance del presente estudio, sólo es posible presentar consideraciones acerca de algunos de los aspectos de la guía, en los que efectivamente se presenta un aporte (FIGURA 3). A continuación se enuncian estos aspectos: recopilación de información existente (revisión bibliográfica y adquisición de mapas topográficos y geológicos); trabajo de campo (estudio vulcanológico general, estudio estratigráfico, recolección de muestras); levantamiento de la cartografía geológica (geología de los afloramientos, geometría de los contactos y las estructuras, alteraciones, manifestaciones termales); estudio estructural de detalle (consideraciones sobre permeabilidad, densidad y características de las fracturas); trabajo de laboratorio (descripción de muestras, análisis petrográficos, análisis químicos); resultados e interpretación (existencia y posible ubicación de la fuente de calor, condiciones estratigráficas y estructurales para acumulación de fluidos geotérmicos en el reservorio, existencia de condiciones para la recarga del reservorio); elaboración de un modelo conceptual (descripción de aspectos del sistema geotérmico, litología del reservorio, unidades que favorecen la circulación de fluidos en el reservorio).

La revisión de información y adquisición de imágenes incluyó: 1) base topográfica a escala 1:100.000 (IGAC) e imágenes satelitales (ASTER GDEM 2);
2) mapa geológico a escala 1:100.000 (Velásquez y Parra, 2002); y mapa geológico a escala 1:50.000 (Perdomo et al., 1986); 3) reportes y estudios acerca de las manifestaciones termales; 4) otros datos del subsuelo (columnas estratigráficas, registros varios) y 5) datos publicados o disponibles sobre la hidrología e hidrogeología.

Se siguió la metodología para levantamiento geológico de Coe et al. (2010) y se enfatizó en la corroboración de litologías y adquisición de datos de detalle en los sitios. Se realizó una visita de campo durante los días septiembre 21-25 de 2015, y el trabajo incluyó la toma de datos geológicos de superficie en 21 afloramientos ubicados principalmente sobre la vía que comunica a Tufiño con Maldonado y en el sitio conocido como Aguas Hediondas (todos los sitios en Ecuador) (FIGURA 1). En los afloramientos rocosos se enfatizó la identificación de rasgos como el estado de alteración de las rocas y su condición de humedad, se tuvo en cuenta la intensidad, origen (térmico o estructural), patrones de orientación, dimensiones y espaciamiento de las diaclasas y fracturas observadas. En el caso de depósitos, se identificó su posible origen teniendo en cuenta, tanto las estructuras como la naturaleza de los clastos y la matriz, y se observaron las características de la matriz para cualificar su permeabilidad (FIGURAS 4 y 5 ).

\section{Tratamiento de muestras}

Se seleccionaron cuatro muestras de rocas frescas (lavas) para su respectiva descripción macroscópica, petrográfica y geoquímica elemental en roca total.

Las muestras previamente partidas se trituraron en un molino de bolas de ágata, el material pulverizado fue secado y se generaron pastillas prensadas de $37 \mathrm{~mm}$ de diámetro para análisis de química de roca total (elementos mayores y traza) por el método de Fluorescencia de Rayos X (FRX). Para un análisis FRX semicuantitativo se utilizó el software SemiQ, realizando 11 barridos para detectar todos los elementos presentes en la muestra, excluyendo $\mathrm{H}, \mathrm{C}, \mathrm{Li}, \mathrm{Be}, \mathrm{B}, \mathrm{N}, \mathrm{O}$ y los elementos transuránicos. La información de los análisis fue procesada usando las rutinas incluidas en GeoChemical Data toolkit-GCDkit versión 4.1 (Janoušek et al., 2006). La descripción y clasificación petrográfica se realizó en las secciones delgadas correspondientes mediante la técnica de conteo de puntos en cinco campos visuales seleccionados, determinando en cada campo visual los porcentajes de vidrio, microlitos y fenocristales, así como los porcentajes de minerales esenciales y secundarios: Plagioclasas, olivinos, piroxenos y opacos. 


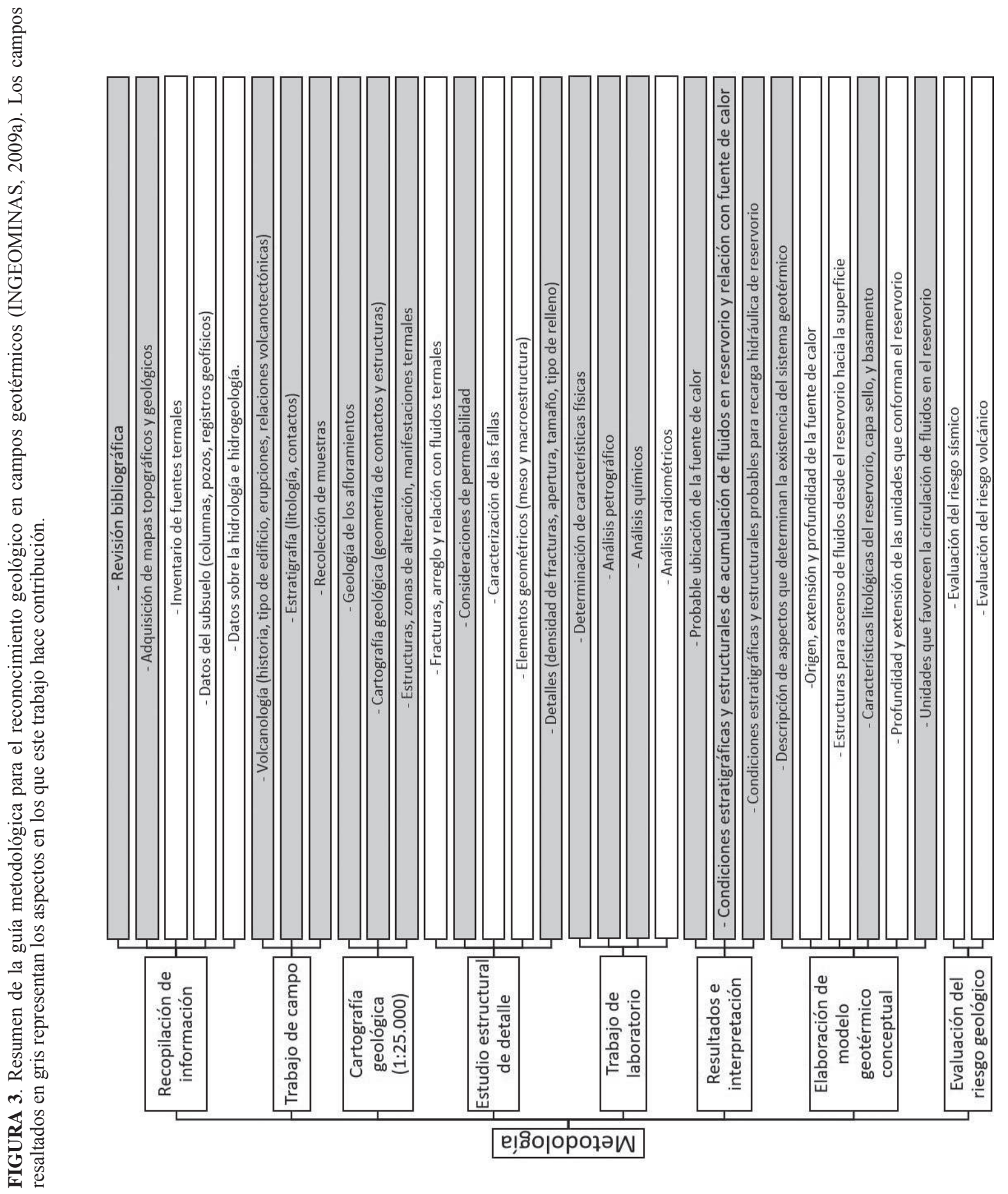



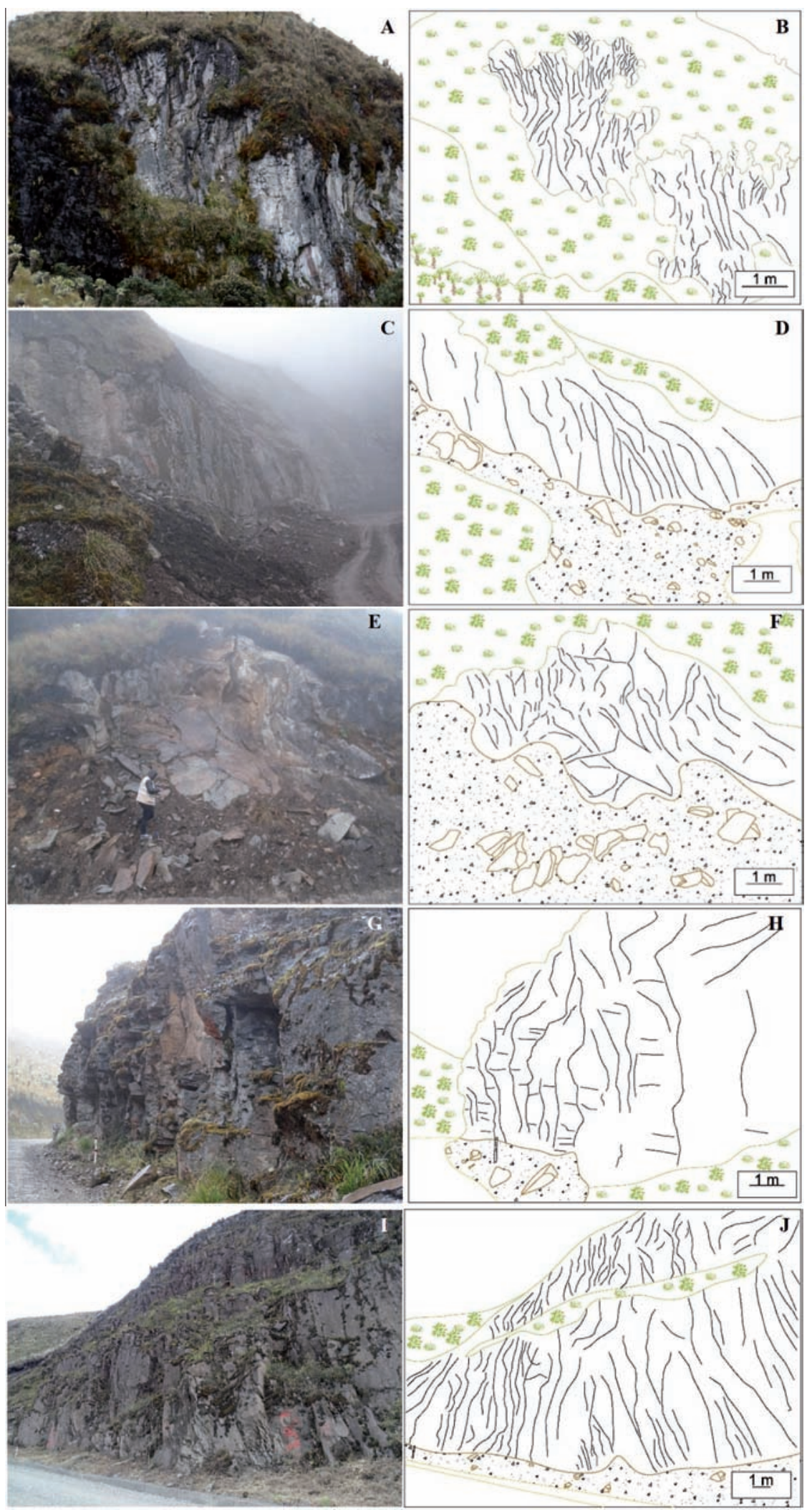

FIGURA 4. Fotos y dibujos esquemáticos de afloramientos rocosos. Las líneas negras resaltan las dimensiones y configuraciones de estructuras (fallas, diaclasas, fracturas) y las líneas de color café representan contactos. A y B. Estación 008. C y D. Estación 010. E y F. Estación 012. G y H. Estación 018. I y J. Estación 020. 

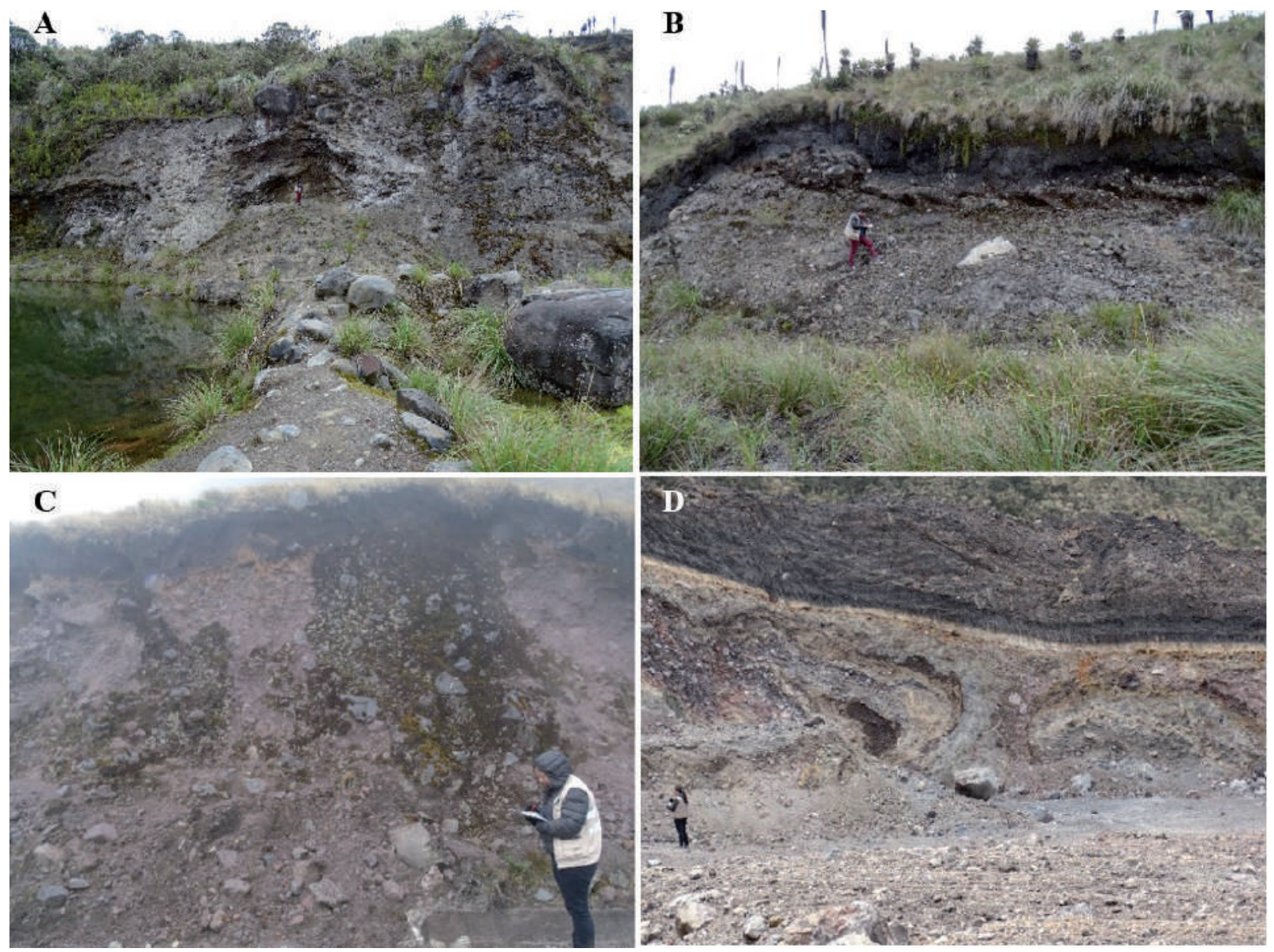

FIGURA 5. Fotos de afloramientos de depósitos. A. Posible depósito de lahar. B. Depósito glaciar (morrena). C. Depósito de talus. D. Depósito de corriente de densidad piroclástica.

La identificación del tipo de plagioclasa se realizó con base en las medidas de ángulos de extinción del método de Michel-Levy.

Los análisis de petrografía y geoquímica (FIGURAS 6 y 7) fueron comparados con la información bibliográfica sobre geología consignada en la cartografía geológica de mayor resolución disponible, a escala 1:50.000 (Perdomo et al., 1986), la cual contiene además datos sobre los productos explosivos y efusivos, rasgos estructurales, zonas de alteración y manifestaciones termales.

\section{Caracterización geomecánica}

Para el estudio estructural y de caracterización geomecánica cualitativa y semicuantitativa de cinco sitios, se utilizó el sistema de clasificación de macizos rocosos (RMR-Rock Mass Rating) (Bieniawski, 1989; Jerram y Petford, 2011) el cual usa cinco parámetros para identificar las características geotécnicas de la roca: 1) resistencia de la roca intacta bajo compresión uniaxial; 2) porcentaje de calidad de la roca $(\% R Q D-$ Rock Quality Designation); 3) espaciamiento de discontinuidades; 4) condición de las discontinuidades y 5) condición general de agua. A continuación, se describe paso a paso el procedimiento para la clasificación de macizos rocosos.

Paso 1: Asignar valores para los cinco parámetros mencionados, de acuerdo a los criterios de la TABLA 2. Dependiendo del tipo de roca, y usando valores predeterminados (Hoek y Brown, 1997), se asigna un valor al parámetro 1. 
TABLA 2. Criterios del sistema de clasificación de un macizo rocoso (RMR). Traducido y modificado de Bieniawski (1989).

\begin{tabular}{|c|c|c|c|c|c|c|c|}
\hline $\begin{array}{l}\text { 1. Resistencia de la roca intacta } \\
\text { a la compresión uniaxial (MPa) }\end{array}$ & $>250$ & $100-250$ & $50-100$ & $25-50$ & $5-25$ & $1-5$ & $<1$ \\
\hline Valoración & 15 & 12 & 7 & 4 & 2 & 1 & 0 \\
\hline 2. \% RQD & $90-100$ & $75-90$ & & $50-75$ & $25-50$ & & $<25$ \\
\hline Valoración & 20 & 17 & & 13 & 8 & & 3 \\
\hline $\begin{array}{l}\text { 3. Espaciamiento entre } \\
\text { discontinuidades (m) }\end{array}$ & $>2$ & $0,6-2$ & & $0,2-0,6$ & $0,06-0,2$ & & $<0,06$ \\
\hline Valoración & 20 & 15 & & 10 & 8 & & 5 \\
\hline $\begin{array}{l}\text { 4. Condición de las } \\
\text { discontinuidades }\end{array}$ & $\begin{array}{c}\text { Muy rugosa, } \\
\text { segmentaciones, } \\
\text { no separación, sin } \\
\text { erosión }\end{array}$ & $\begin{array}{l}\text { Paredes } \\
\text { rugosas, } \\
\text { separación } \\
<0,1 \mathrm{~mm} \text {, } \\
\text { algo de } \\
\text { meteorización }\end{array}$ & & $\begin{array}{l}\text { Levemente } \\
\text { rugosas, } \\
\text { separación } \\
<1 \mathrm{~mm}, \\
\text { altamente } \\
\text { meteorizada }\end{array}$ & $\begin{array}{l}\text { Caras } \\
\text { suaves o } \\
\text { salbanda } \\
\text { con }<5 \mathrm{~mm} \\
\text { espesor, o } \\
\text { separación } \\
\text { uniforme de } \\
1-5 \mathrm{~mm}\end{array}$ & & $\begin{array}{l}\text { Salbanda suave } \\
\text { con espesor } \\
>5 \mathrm{~mm} \text {, o } \\
\text { separación } \\
\text { uniforme }> \\
5 \mathrm{~mm} \text {, roca } \\
\text { encajante } \\
\text { descompuesta }\end{array}$ \\
\hline Valoración & 30 & 25 & & 20 & 10 & & 0 \\
\hline $\begin{array}{l}\text { 5. Condiciones generales de } \\
\text { agua }\end{array}$ & $\begin{array}{l}\text { Completamente } \\
\text { seca }\end{array}$ & Húmeda & & Mojada & Empapada & & Fluida \\
\hline Valoración & 15 & 10 & & 7 & 4 & & 0 \\
\hline
\end{tabular}

Para obtener valores del parámetro 2, se usa la fórmula:

$$
\% R Q D=\frac{\left.\sum \text { (longitud de bloques continuos de roca en línea de referencia, }>0,1 \mathrm{~m}\right)}{\text { longitud línea de referencia }(1,0 \mathrm{~m})} \times 100
$$

donde el numerador es la suma de las separaciones mayores a $0,1 \mathrm{~m}(10 \mathrm{~cm})$ entre discontinuidades que intersectan una línea horizontal de referencia en el afloramiento. En la ecuación 1 debe tenerse presente que, si la longitud de la línea de referencia cambia, también debe actualizarse la condición del numerador para mantener las proporciones. Por ejemplo, si la longitud de línea de referencia es $10 \mathrm{~m}$, entonces la condición del numerador ahora es "> 1 m" $(100 \mathrm{~cm})$.

Para la valoración del parámetro 3 se usa la mediana de los valores observados de separaciones entre discontinuidades, luego de descartar separaciones de acuerdo a la condición del numerador en la ecuación 1. Se continúa con la evaluación de los parámetros 4 y 5. Al final se obtendrán cinco valoraciones individuales cuya suma, el valor $R M R$, será $\leq 100$.

Paso 2: Estimar la resistencia de la masa rocosa. El método usa el valor $R M R$ y la relación empírica entre resistencia de las rocas y los valores relativos entre los esfuerzos principales durante la fractura (Hoek y Brown, 1982). El proceso involucra el cálculo de dos parámetros Hoek-Brown: $m$ y $s$, usando las relaciones:

$$
m=m_{i} e^{\frac{(R M R-100)}{28}}
$$

$$
S=e^{\frac{(R M R-100)}{9}}
$$

donde $m_{i}=25 \pm 5$ (Brown, 1981; Jerram y Petford, 2011). Estos parámetros son usados para la determinación de dos de las principales propiedades mecánicas y geotécnicas de una masa rocosa, a saber: resistencia compresiva del macizo rocoso $\left(\sigma_{c m}\right)$ y resistencia tensional del macizo rocoso $\left(\sigma_{t m}\right)$, en las fórmulas:

$$
\begin{gathered}
\sigma_{c m}=\left(s \sigma_{c}^{2}\right)^{1 / 2} \\
\sigma_{t m}=\frac{\sigma_{c}}{2}\left[m-\left(m^{2}+4 s\right)^{1 / 2}\right]
\end{gathered}
$$

donde $\sigma_{c}$ es la resistencia compresiva de la roca intacta, que para el caso de andesitas se toma con un valor de $128 \mathrm{MPa}$ (Brown, 1981).

Adicionalmente se realizó el reconocimiento de fuentes termales, manantiales, lagunas y solfataras que permitieron cualificar la permeabilidad de las rocas y los depósitos como insumos adicionales para el planteamiento del modelo conceptual geotérmico. 

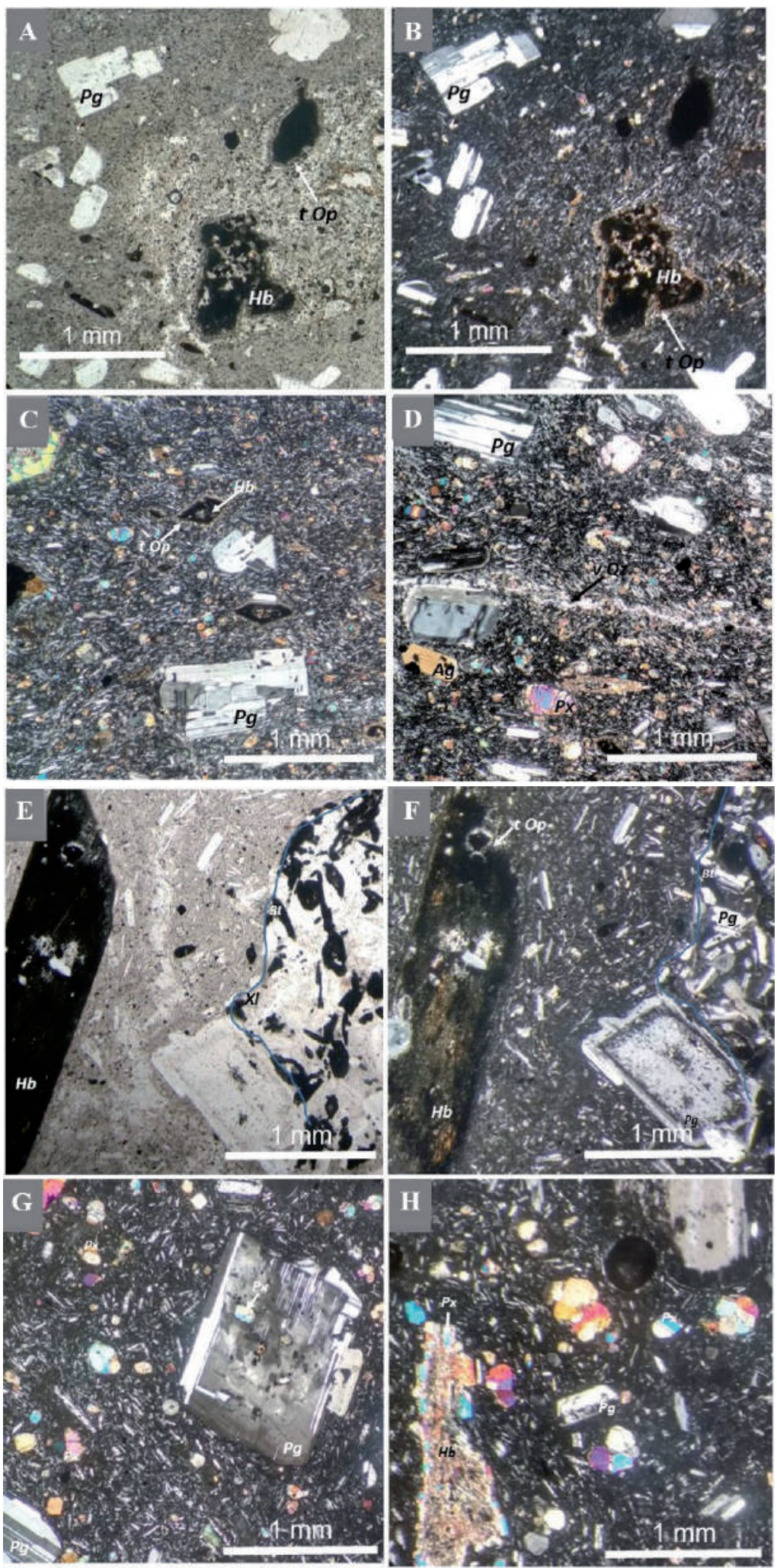

FIGURA 6. Microfotografías de campos visuales seleccionados por muestra. A y B. Muestra M-005 PPL y XPL, respectivamente (aumento 4X). C y D. Muestra M-006, dos campos visuales, XPL (4X). E y F. Muestra M-007 PPL y XPL, respectivamente (aumento 4X). G y H. Muestra M-008, dos campos visuales, XPL (4X). Pg: Plagioclasa; Hb: Hornblenda; Cpx: Clinopiroxeno; Opx: Ortopiroxeno; Ag: Augita titanífera; Bt: Biotita; vQz: Venilla de cuarzo; tOp: Textura de opacitización. 

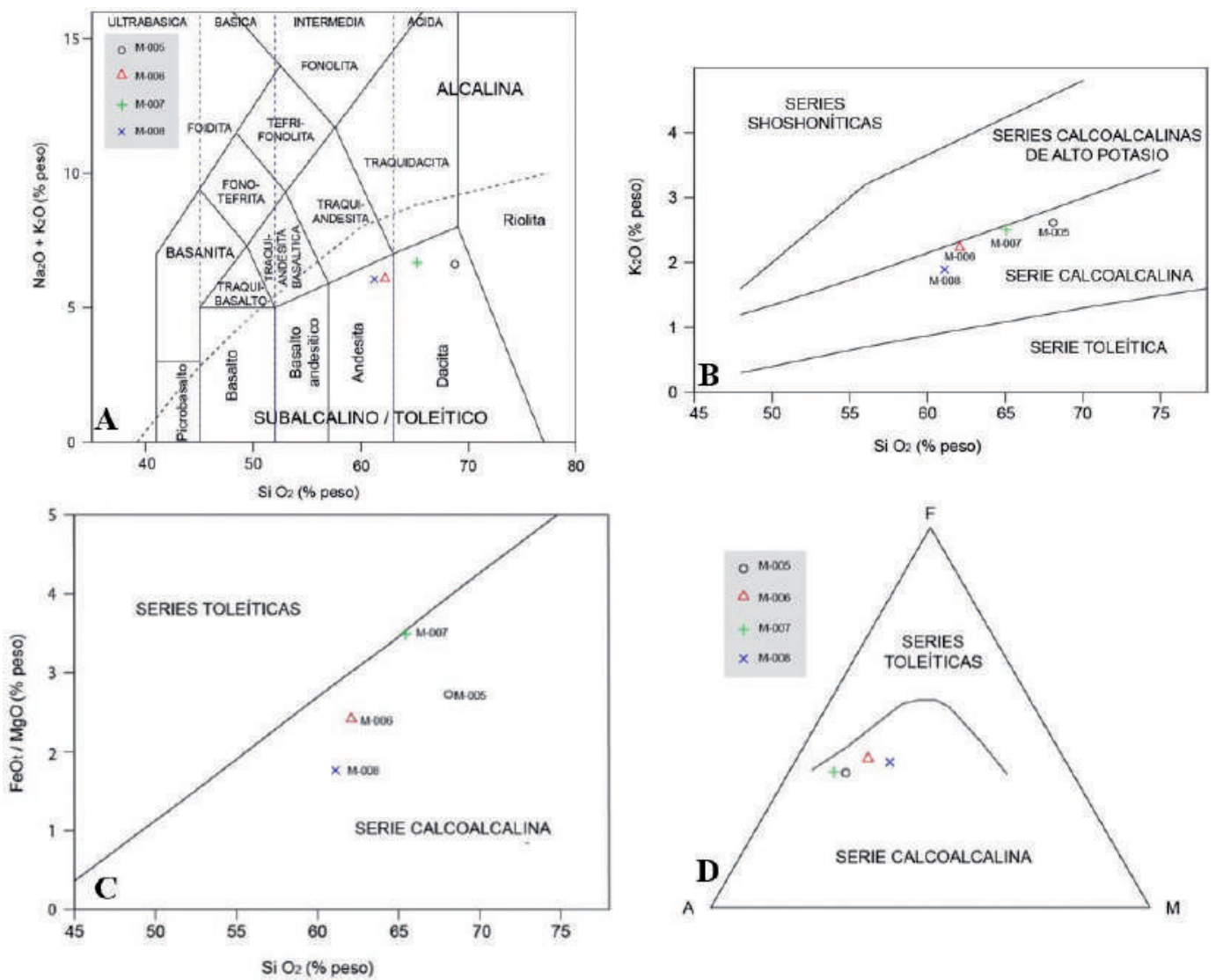

FIGURA 7. Diagramas de variación y discriminación para las muestras analizadas del CVCCN. A. Diagrama de variación y clasificación geoquímica $\mathrm{Na}_{2} \mathrm{O}+\mathrm{K}_{2} \mathrm{O}$ vs $\mathrm{SiO}_{2}$ (Le Bas et al., 1986). B. Diagrama $\mathrm{K}_{2} \mathrm{O}$ vs $\mathrm{SiO}_{2}$ de discriminación de las series de toleitica a shoshonitica (Peccerillo y Taylor, 1976). C. Diagrama $\mathrm{SiO}_{2}-\mathrm{FeO}_{\mathrm{t}} / \mathrm{MgO}$ de discriminación de las series toleítica y calcoalcalina (Miyashiro, 1974). D. Diagrama AFM (Irvine y Baragar, 1971).

\section{RESULTADOS}

Los análisis químicos, petrográficos, y de mediciones en campo brindan resultados de detalle en relación a las dos subseciones previamente presentadas en la Metodología: levantamiento geológico y caracterización geomecánica de los sitios visitados. A continuación, se presentan dichos resultados.

\section{Levantamiento geológico}

Se inspeccionaron 21 afloramientos de rocas y depósitos, todos ubicados dentro o en las inmediaciones del área de interés del Proyecto Geotérmico Binacional (FIGURA 1). Todos los afloramientos rocosos corresponden a lavas (FIGURA 4) y los afloramientos de materiales no litificados incluyen depósitos de sedimento glacial (morrenas), depósitos volcaniclásticos (lahares y flujos piroclásticos) y depósitos de talus (FIGURA 5). Los resultados de observaciones en los afloramientos se presentan en la
TABLA 3. De las nueve muestras de rocas recolectadas se prepararon cuatro (codificadas como M-005, M-006, M-007, y M-008) para realización de análisis petrográficos en secciones delgadas y correspondientes análisis químicos (ver sección Metodología).

\section{Análisis petrográficos}

Las cuatro muestras analizadas, clasificadas como andesitas (Streckeisen, 1973), exhiben los siguientes rasgos comunes: 1) Carácter hipocristalino o hipohialino y textura microporfirítica con contenido moderado a bajo de fenocristales (principalmente inequigranulares y con formas anhedrales o suhedrales) y dominio de matriz mixta con textura fluida; 2) Mineralogía principalmente de plagioclasa andesina, augita, biotita, hornblenda y opacos; 3) Opacitización en hornblendas o biotitas (FIGURA 6). La TABLA 4 resume los resultados del análisis petrográfico y la correspondiente clasificación. 
TABLA 3. Resumen de las características de los afloramientos visitados en el área de estudio. La FIGURA 1 muestra la ubicación de las estaciones.

\begin{tabular}{|c|c|c|c|c|c|c|c|}
\hline Est. & Lat. $\left(^{\circ}\right)$ & $\operatorname{Lon}\left({ }^{\circ}\right)$ & $\begin{array}{c}\text { Altura } \\
\text { (m.s.n.m.) }\end{array}$ & Litología & Permeabilidad & Observaciones & Muestra \\
\hline 1 & 0,79773 & $-77,86333$ & 3327 & Lavas & Moderada & Diaclasas de origen térmico. & M-001 \\
\hline 2 & 0,80819 & $-77,89032$ & 3538 & $\begin{array}{l}\text { Depósito de } \\
\text { lahar }\end{array}$ & Moderada & $\begin{array}{l}\text { Fracturas métricas, laguna con } \\
\text { agua a } \mathrm{T}^{\circ} \text { por encima de ambiente, } \\
\text { profundidad } 1,5 \mathrm{~m} \text {. }\end{array}$ & M-002 \\
\hline 3 & 0,81011 & $-77,90278$ & 3565 & $\begin{array}{l}\text { Depósito de } \\
\text { lahar }\end{array}$ & Moderada & $\begin{array}{l}\text { Cristales aciculares de color naranja y } \\
\text { ocre, alteración hidrotermal. A } 300 \mathrm{~m} \\
\text { en dirección NW acantilado de lavas } \\
\text { con apariencia estratificada, fracturas } \\
\text { métricas abiertas. }\end{array}$ & $\begin{array}{l}\text { M-003 } \\
\text { M-004 }\end{array}$ \\
\hline 4 & 0,8098 & $-77,9058$ & 3596 & Lavas & Alta & $\begin{array}{l}\text { Fracturas, alteración hidrotermal, } \\
\text { diaclasas con estructura en rampa. } \\
\text { Termales "Aguas Hediondas", } \mathrm{T}^{\circ} \\
\sim 59^{\circ} \text {, alteración hidrotermal, pátina de } \\
\text { azufre, solfataras con cristalización de } \\
\text { azufre. Muestra de mano con carácter } \\
\text { leucocrático. }\end{array}$ & M-005 \\
\hline 5 & 0,7819 & $-77,89356$ & 3620 & Till & Alta & $\begin{array}{l}\text { Masivo, alto contenido de materia } \\
\text { orgánica, paralelo a un valle glaciar de } \\
\text { orden mayor, morfología glaciar. }\end{array}$ & \\
\hline 6 & 0,79791 & $-77,91267$ & 3893 & $\begin{array}{l}\text { Depósitos no } \\
\text { consolidados } \\
\text { suprayacidos } \\
\text { por capa de } \\
\text { ceniza y suelo }\end{array}$ & Alta & $\begin{array}{l}\text { Estructura estratificada incipiente } \\
\text { (capas, gradación). A } 50 \mathrm{~m} \text { al E afloran } \\
\text { lavas muy diaclasadas, se midió el } \\
\text { dato de una familia con dirección } \\
\text { N25E/72NW. }\end{array}$ & \\
\hline 7 & 0,79871 & $-77,91409$ & 3894 & lavas & Alta & $\begin{array}{l}\text { Diaclasas con dirección N10W/65SE, } \\
\text { fracturas métricas buzando al SE. } \\
\text { En algunas paredes de diaclasas } \\
\text { se aprecian estructuras lineales no } \\
\text { identificadas. }\end{array}$ & \\
\hline 8 & 0,79718 & $-77,9197$ & 3933 & Lavas & Alta & $\begin{array}{l}\text { Diaclasas con dirección N54E/15SW, } \\
\text { intenso diaclasamiento con múltiples } \\
\text { orientaciones, se observa manantial de } \\
\text { agua fría a la base. Muestra de mano } \\
\text { con carácter leucocrático. }\end{array}$ & M-006 \\
\hline 9 & 0,79999 & $-77,92624$ & 3995 & $\begin{array}{l}\text { Depósitos no } \\
\text { consolidados }\end{array}$ & Baja & $\begin{array}{l}\text { Posible depósito de movimiento en } \\
\text { masa (derrubio, deslizamiento de } \\
\text { rocas, talus) o sedimento glaciar. }\end{array}$ & \\
\hline 10 & 0,80011 & $-77,93085$ & 4006 & Lavas & Baja & $\begin{array}{l}\text { Diaclasas métricas, verticales con } \\
\text { dirección SE. }\end{array}$ & \\
\hline 11 & 0,79744 & $-77,9224$ & 3961 & Lavas & Alta & $\begin{array}{l}\text { Diaclasas con múltiples orientaciones, } \\
\text { apariencia prismática en los bloques. }\end{array}$ & \\
\hline 12 & 0,79837 & $-77,93461$ & 4026 & Lavas & Moderada & $\begin{array}{l}\text { Masivas. Diaclasas con aperturas } \\
\text { milimétricas, fracturas métricas de } \\
\text { aperturas milimétricas a centimétricas. } \\
\text { Muestra de mano con carácter } \\
\text { leucocrático. }\end{array}$ & M-007 \\
\hline 13 & 0,80173 & $-77,93682$ & 4028 & $\begin{array}{l}\text { Depósito de } \\
\text { talus }\end{array}$ & Alta & $\begin{array}{l}\text { Posible depósito de talus, clasto } \\
\text { soportado y en algunas secciones } \\
\text { matriz soportado. }\end{array}$ & \\
\hline 14 & 0,80056 & $-77,94476$ & 4079 & Lavas & Alta & $\begin{array}{l}\text { Muy alteradas y diaclasadas, algunas } \\
\text { familias de diaclasas (subhorizontales) } \\
\text { con superficies de oxidación que } \\
\text { reducen la permeabilidad. En contacto } \\
\text { discordante con depósitos de talus y de } \\
\text { caída. }\end{array}$ & \\
\hline
\end{tabular}


Yohanna K. García, John J. Sánchez

Continuación TABLA 3.

\begin{tabular}{|c|c|c|c|c|c|c|c|}
\hline Est. & Lat. $\left(^{\circ}\right)$ & $\operatorname{Lon}\left({ }^{\circ}\right)$ & $\begin{array}{c}\text { Altura } \\
\text { (m.s.n.m.) }\end{array}$ & Litología & Permeabilidad & Observaciones & Muestra \\
\hline 15 & 0,79828 & $-77,94846$ & 4053 & Lavas & Moderada & $\begin{array}{l}\text { Intenso diaclasamiento, posible } \\
\text { cataclasis, alteración hidrotermal } \\
\text { (silícea). Debido al estado de } \\
\text { diaclasamiento la permeabilidad debe } \\
\text { ser alta pero la alteración silícea puede } \\
\text { reducirla. }\end{array}$ & \\
\hline 16 & 0,7976 & $-77,94959$ & 3999 & Lavas & Alta & $\begin{array}{l}\text { Intenso diaclasamiento, dos familias } \\
\text { dominantes con direcciones } \\
\text { N55W/50NE y N36E/57SE que } \\
\text { producen patrones rómbicos y } \\
\text { prismáticos en los bloques de diferentes } \\
\text { tamaños, el diaclasamiento favorece la } \\
\text { meteorización esferoidal. }\end{array}$ & \\
\hline 17 & 0,80554 & $-77,95696$ & 3921 & Lavas & Moderada a alta & $\begin{array}{l}\text { Diaclasamiento intenso. Familia de } \\
\text { diaclasas verticales con orientación } \\
\text { N24E. }\end{array}$ & \\
\hline 18 & 0,80717 & $-77,95487$ & 3914 & Lavas & Moderada & $\begin{array}{l}\text { Disyunción columnar. Diaclasamiento } \\
\text { térmico dominante (patrón normal, se } \\
\text { intensifica de base a techo). Lavas con } \\
\text { textura porfirítica fina a media (olivinos } \\
\text { alterados, piroxenos y plagioclasas). } \\
\text { Presentan alteración hidrotermal, } \\
\text { con cristales aciculares de colores } \\
\text { anaranjados a ocres. Muestra de mano } \\
\text { con carácter leucocrático. }\end{array}$ & M-008 \\
\hline 19 & 0,80879 & $-77,96634$ & 3725 & $\begin{array}{l}\text { Depósito } \\
\text { de flujo } \\
\text { piroclastico }\end{array}$ & Alta & $\begin{array}{l}\text { Granulometría variable (cm-m) } \\
\text { principalmente lavas y escorias } \\
\text { basálticas, en contacto con depósitos } \\
\text { de caída y suelos ricos en materia } \\
\text { orgánica muy agrietados, grietas } \\
\text { perpendiculares a la morfología } \\
\text { algunas afectando incluso el depósito. } \\
\text { Posible fuente: Volcán Cerro Negro. } \\
\text { Se observan manantiales cercanos. Las } \\
\text { unidades de flujo piroclástico presentan } \\
\text { morfología de depósito de canal. }\end{array}$ & \\
\hline 20 & 0,80857 & $-77,99953$ & 3535 & Lavas & Moderada a alta & $\begin{array}{l}\text { Diaclasamiento intenso con aperturas } \\
\text { milimétricas. Las diaclasas térmicas } \\
\text { están verticalizadas. }\end{array}$ & M-009 \\
\hline 21 & 0,8068 & $-77,9814$ & 3674 & Lavas & Alta & $\begin{array}{l}\text { Lavas muy alteradas y diaclasadas } \\
\text { en contacto discordante con posible } \\
\text { depósito epiclástico (material } \\
\text { retrabajado, con clastos redondeados } \\
\text { estratificación incipiente) suprayacido } \\
\text { por depósitos de caída y suelos. }\end{array}$ & \\
\hline
\end{tabular}

\section{Análisis geoquímicos}

Las muestras analizadas del CVCCN presentan un contenido de $\mathrm{SiO}_{2}$ entre $61,12 \%$ y $68,08 \%$ en peso (TABLA 5), que corresponden a composiciones intermedias a félsicas. El valor más bajo del rango corresponde a la muestra M-008 y el valor más alto a la muestra M-005.

El diagrama de variación $\left(\mathrm{Na}_{2} \mathrm{O}+\mathrm{K}_{2} \mathrm{O}\right.$ vs $\left.\mathrm{SiO}_{2}\right)$ confirma la clasificación modal QAPF (Streckeisen,
1973) de las muestras M-006 y M-007 como andesitas y se observa que las muestras M-005 y M-007 corresponden a dacitas (FIGURA 7A). Los diagramas de discriminación $\left(\mathrm{K}_{2} \mathrm{O}\right.$ vs $\mathrm{SiO}_{2}, \mathrm{FeO}_{\mathrm{t}} / \mathrm{MgO}$ vs $\mathrm{SiO}_{2}$, y AFM) ubican las muestras en la serie calcoalcalina de magmas. Teniendo en cuenta la estrecha relación que hay entre el ambiente geotectónico y las series de magmas, se confirma el ambiente tectónico de zona de subducción y arco continental para estas lavas. 


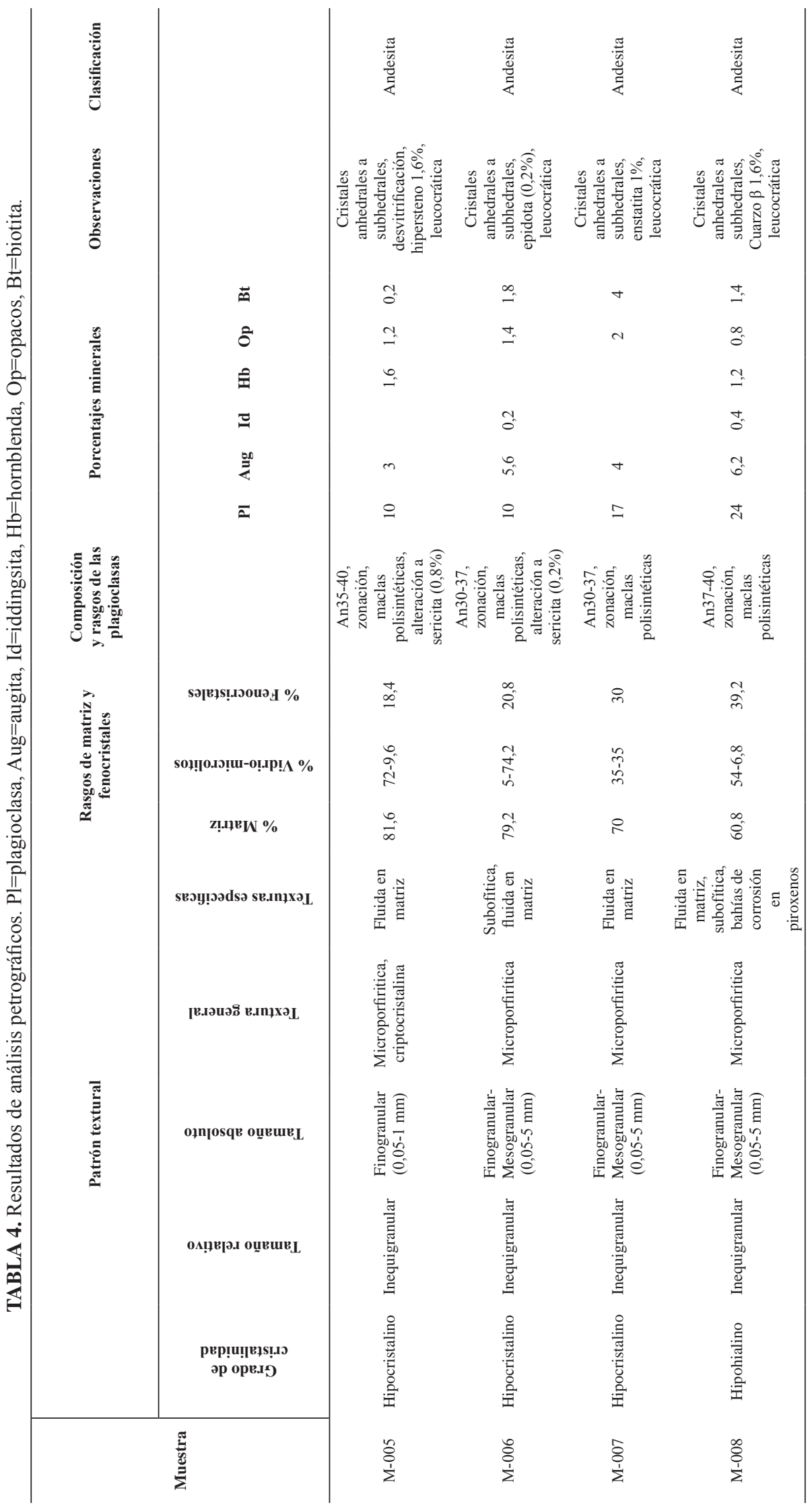


TABLA 5. Datos de elementos mayores (\% en peso) y traza (ppm) de las muestras del CVCCN.

\begin{tabular}{|c|c|c|c|c|}
\hline $\begin{array}{c}\text { Elementos mayores } \\
\text { (\% peso) }\end{array}$ & M-005 & M-006 & M-007 & M-008 \\
\hline $\mathrm{SiO}_{2}$ & 68,081 & 62,076 & 65,061 & 61,106 \\
\hline $\mathrm{Al}_{2} \mathrm{O}_{3}$ & 13,268 & 16,870 & 16,268 & 15,742 \\
\hline $\mathrm{Fe}_{2} \mathrm{O}_{3}$ & 5,000 & 5,921 & 4,869 & 6,404 \\
\hline $\mathrm{CaO}$ & 4,191 & 5,721 & 4,882 & 6,287 \\
\hline $\mathrm{Na}_{2} \mathrm{O}$ & 3,933 & 3,842 & 4,049 & 4,150 \\
\hline $\mathrm{K}_{2} \mathrm{O}$ & 2,613 & 2,225 & 2,61 & 1,89 \\
\hline$M g O$ & 1,651 & 2,207 & 1,254 & 3,267 \\
\hline $\mathrm{TiO}_{2}$ & 0,648 & 0,605 & 0,526 & 0,640 \\
\hline $\mathrm{P}_{2} \mathrm{O}_{5}$ & 0,192 & 0,142 & 0,16 & 0,174 \\
\hline $\mathrm{SO}_{3}$ & 0,149 & 0,064 & 0,027 & 0,010 \\
\hline $\mathrm{MnO}$ & 0,075 & 0,102 & 0,086 & 0,108 \\
\hline \multicolumn{5}{|l|}{ Elementos traza (ppm) } \\
\hline$B a$ & 690 & 760 & 770 & 480 \\
\hline$S r$ & 400 & 360 & 410 & 400 \\
\hline $\mathrm{Zr}$ & 200 & 170 & 190 & 160 \\
\hline $\mathrm{Cl}$ & 140 & 280 & 110 & 300 \\
\hline V & 140 & 150 & 220 & 220 \\
\hline $\mathrm{Cr}$ & 110 & 110 & 80 & 330 \\
\hline Co & 90 & 70 & 70 & 90 \\
\hline$R b$ & 90 & 80 & 90 & 50 \\
\hline$Z n$ & 60 & 80 & 70 & 80 \\
\hline $\mathrm{Cu}$ & 50 & 110 & 50 & 70 \\
\hline$Y$ & 30 & 40 & 30 & 20 \\
\hline $\mathrm{Pb}$ & 0 & 60 & 0 & 0 \\
\hline
\end{tabular}

\section{Análisis de estructuras y propiedades geotécnicas en afloramientos rocosos}

En todos los afloramientos rocosos se observaron discontinuidades clasificables como diaclasas (tanto térmicas como debidas a otros esfuerzos), y fracturas, con diferentes orientaciones, inclinaciones, espaciamientos, y condiciones de relleno (FIGURA 4). En varios sitios, dependiendo de las condiciones de acceso, la inspección visual permitió corroborar la superposición de los diferentes efectos o el dominio de un tipo de deformación sobre otro. Los efectos de la meteorización química y física, la cubierta vegetal y procesos superficiales de remoción en masa enmascaraban comúnmente los rasgos primarios de interés. También se encontraron evidencias de alteraciones hidrotermales en grados variables de avance, principalmente en los alrededores de las fuentes termales, y variabilidad en las condiciones de humedad de los sitios incluyendo en algunos casos manantiales fríos en zonas rocosas y de depósitos (TABLA 3). Se midieron espaciamientos entre discontinuidades en el rango $20 \mathrm{~cm}-80 \mathrm{~cm}$, con aperturas en el rango $0,1 \mathrm{~mm}-$ $15 \mathrm{~mm}$, en condiciones de roca húmeda principalmente. Se asignaron valores según los criterios del sistema $R M R$ (Bieniawski, 1989; TABLA 2) en cinco sitios (E8, E10, E12, E18, y E20) en los cuales la resistencia compresiva de la roca intacta fue tomada con valor uniforme de $128 \mathrm{MPa}$ (andesitas en todos los casos), dando como resultado valores de $\% R Q D$ en el rango $90 \%-96 \%$ (TABLA 6). En conjunto, se encontró que el valor $R M R$ de cinco sitios varía en el rango 52 - 77 (TABLA 7). De acuerdo a lo anterior, se calcularon valores de resistencia compresiva y tensional de los cinco afloramientos que varían en los rangos $8,89 \leq \sigma_{c m} \leq 35,67$ y $-1,13 \leq \sigma_{t m} \leq$ $-0,11$, respectivamente (TABLA 8 ). 
TABLA 6. Características de las rocas y sus discontinuidades en cinco sitios seleccionados.

\begin{tabular}{|c|c|c|c|c|c|}
\hline $\begin{array}{c}\text { Parámetros de clasificación de un macizo } \\
\text { rocoso (RMR) }\end{array}$ & $\begin{array}{l}\text { Estación } \\
008\end{array}$ & $\begin{array}{l}\text { Estación } \\
010\end{array}$ & $\begin{array}{l}\text { Estación } \\
012\end{array}$ & $\begin{array}{l}\text { Estación } \\
018\end{array}$ & $\begin{array}{l}\text { Estación } \\
020\end{array}$ \\
\hline 1. Resistencia de la roca $(\mathrm{MPa})^{*}$ & 128 & 128 & 128 & 128 & 128 \\
\hline 2. \% RQD & $90 \%$ & $92 \%$ & $93 \%$ & $96 \%$ & $90 \%$ \\
\hline 3. Espaciamiento entre discontinuidades $(\mathrm{m})^{* *}$ & 0,2 & 0,4 & 0,5 & 0,8 & 0,6 \\
\hline 4. Condición de discontinuidades & $\begin{array}{l}\text { Separación } \\
\quad<1 \mathrm{~mm}\end{array}$ & $\begin{array}{l}\text { Separación } \\
<0,1 \mathrm{~mm}\end{array}$ & $\begin{array}{l}\text { Separación } \\
5-15 \mathrm{~mm}\end{array}$ & $\begin{array}{l}\text { Separación } \\
\quad<1 \mathrm{~mm}\end{array}$ & $\begin{array}{l}\text { Separación } \\
<1 \mathrm{~mm}\end{array}$ \\
\hline 5. Condiciones generales de agua & $\begin{array}{c}\text { Roca } \\
\text { empapada }\end{array}$ & $\begin{array}{l}\text { Roca } \\
\text { húmeda }\end{array}$ & $\begin{array}{c}\text { Roca } \\
\text { húmeda }\end{array}$ & $\begin{array}{c}\text { Roca } \\
\text { húmeda }\end{array}$ & $\begin{array}{c}\text { Roca } \\
\text { húmeda }\end{array}$ \\
\hline
\end{tabular}

*Valores uniformes (Hoek y Brown, 1997) para todas las muestras relacionadas, correspondientes a andesitas.

**Se reporta el valor de la mediana (como medida central) de los espaciamientos entre discontinuidades, una vez descartados los valores inferiores a $10 \mathrm{~cm}$.

TABLA 7. Valoraciones para clasificación $R M R$ de los cinco afloramientos.

\begin{tabular}{|c|c|c|c|c|c|}
\hline Parámetro* & Estación 008 & Estación 010 & Estación 012 & Estación 018 & Estación 020 \\
\hline 1 & 12 & 12 & 12 & 12 & 12 \\
\hline 2 & 17 & 20 & 20 & 20 & 17 \\
\hline 3 & 8 & 10 & 10 & 15 & 10 \\
\hline 4 & 20 & 25 & 0 & 20 & 20 \\
\hline 5 & 4 & 10 & 10 & 10 & 10 \\
\hline$\Sigma=R M R \rightarrow$ & 61 & 77 & 52 & 77 & 69 \\
\hline
\end{tabular}

*De acuerdo a número en la primera columna de las TABLAS 2 y 6.

TABLA 8. Valores de las principales propiedades mecánicas y geotécnicas de los cinco afloramientos.

\begin{tabular}{cccccc}
\hline Parámetro & $\begin{array}{c}\text { Estación } \\
\mathbf{0 0 8}\end{array}$ & $\begin{array}{c}\text { Estación } \\
\mathbf{0 1 0}\end{array}$ & $\begin{array}{c}\text { Estación } \\
\mathbf{0 1 2}\end{array}$ & $\begin{array}{c}\text { Estación } \\
\mathbf{0 1 8}\end{array}$ & $\begin{array}{c}\text { Estación } \\
\mathbf{0 2 0}\end{array}$ \\
\hline $\boldsymbol{m}_{\min }{ }^{*}$ & 4,97 & 8,80 & 3,60 & 8,80 & 6,61 \\
$\boldsymbol{m}_{\max } * *$ & 7,45 & 13,19 & 5,40 & 13,19 & 9,92 \\
$\boldsymbol{m}_{\boldsymbol{a v g}} * *$ & 6,21 & 11,00 & 4,50 & 11,00 & 8,26 \\
$\boldsymbol{s}$ & 0,013 & 0,078 & 0,005 & 0,078 & 0,032 \\
$\left(\boldsymbol{\sigma}_{\boldsymbol{c m}}\right), \mathbf{M p a}$ & 14,66 & 35,67 & 8,89 & 35,67 & 22,87 \\
$\left(\boldsymbol{\sigma}_{t m}\right)_{\min }, \mathbf{M p a}$ & $-0,338$ & $-1,129$ & $-0,172$ & $-1,129$ & $-0,618$ \\
$\left(\boldsymbol{\sigma}_{t m}\right)_{\max }, \mathbf{M p a}$ & $-0,225$ & $-0,753$ & $-0,114$ & $-0,753$ & $-0,412$ \\
$\left(\boldsymbol{\sigma}_{t m}\right)_{\mathbf{a v g}}, \mathbf{M p a}$ & $-0,270$ & $-0,903$ & $-0,137$ & $-0,903$ & $-0,494$ \\
\hline
\end{tabular}

$m_{\min }, m_{\max }, m_{\text {avg }}$ : Valores mínimos, máximos y promedios, del parámetro Hoek-Brown, $m$, asumiendo valores extremos y centrales de la variable $m_{i}$ en la ecuación 2.

$\sigma$ : Resistencia compresiva del macizo rocoso calculada con la ecuación 4.

$\left(\sigma_{t m}\right)_{\min },\left(\sigma_{t m}\right)_{\max },\left(\sigma_{t m}\right)_{\text {avg }}$ : Valores mínimos, máximos, y promedios, de resistencia tensional del macizo rocoso calculados con la ecuación 5 , asumiendo valores extremos y centrales de la variable $m_{i}$ en la ecuación 2 . 


\section{Condiciones generales de las aguas: subterráneas, manantiales fríos $y$ manifestaciones hidrotermales}

Durante los recorridos se evaluó la condición general de las aguas en los sitios mediante inspección visual de las discontinuidades en las rocas y documentando la presencia de manantiales fríos y fuentes termales. En las estaciones E8, E10, E12, E18 y E20, el criterio de la condición de aguas subterráneas fue valorado en el rango 4-10, correspondiente a las cualificaciones "roca empapada" y "roca húmeda" (TABLAS 2, 3 y 6). En dos sitios (E8 y E19) se encontraron manantiales fríos, y en la estación E2 se encontró un manantial con agua a temperatura superior a la ambiental. La estación E4 corresponde al sitio conocido como los termales de "Aguas Hediondas", donde las temperaturas reportadas son $46^{\circ} \mathrm{C}$ y $58^{\circ} \mathrm{C}$ (Lesmes et al., 2004; Carrera y Guevara, 2016).

\section{DISCUSIÓN}

La exploración geológica de áreas geotérmicas en Colombia ha contribuido en parte al avance de proyectos para la construcción de plantas de energía geotérmica hasta las etapas actuales de conclusión de estudios de prefactibilidad, como en el caso del Complejo Volcánico Nevado del Ruiz, e inicio de prefactibilidad, como en el CVCCN (Mejía et al., 2014; Alfaro, 2015). En esta última área, uno de los objetivos principales dentro de los estudios complementarios de prefactibilidad es la construcción de un modelo conceptual, del cual se han ubicado en la literatura dos versiones (Beate, 1991; INER, 2013). Desde esa perspectiva, en el presente trabajo se integran elementos relacionados a 1) estudio geológico superficial en el CVCCN para caracterizar algunas unidades litológicas, 2) análisis de estructuras y propiedades geotécnicas en afloramientos rocosos y 3) condiciones generales de aguas subterráneas y superficiales, todos insumos para refinar el modelo conceptual en preliminar.

En términos del mapa de unidades geológicas de mejor resolución disponible a la fecha (Perdomo et al., 1986) fue posible confirmar la litología aflorante, consistente principalmente de emplazamientos de lava de la primera y segunda actividad del VC (episodios 4 y 6 de Cortés y Calvache, 1996) y depósitos de material fragmental de naturaleza variable, incluyendo derrubios, till, lahares y flujos piroclásticos (TABLA 3, FIGURA 1). La mayor parte de los edificios del CVCCN está formada por acumulaciones de lavas que han alcanzado elevaciones importantes, constituyéndose así en las áreas de precipitación y recarga del sistema hidrogeológico regional (FIGURA 8).

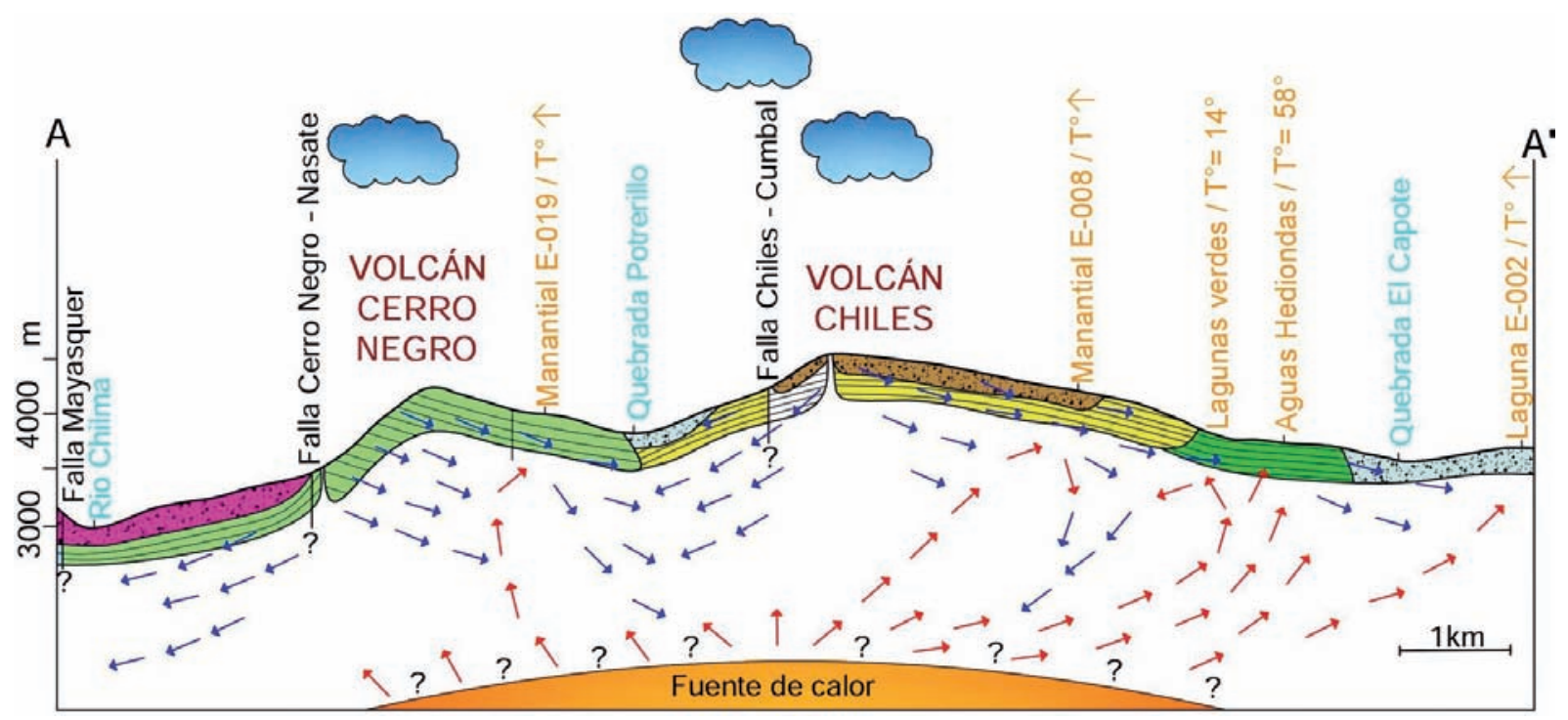

FIGURA 8. Perfil A-A' (ver FIGURA 1) con el modelo geotérmico conceptual propuesto. Nubes: área de recarga. Flechas azules: dirección de flujo del sistema de recarga. Flechas rojas: Dirección de flujo de calor. En naranja: posibles áreas de descarga de aguas termales (se indica temperatura de las aguas cuando se conoce, de lo contrario, se indica temperatura por encima de la temperatura ambiente como " $\mathrm{T}$ o ”. Convención de colores en litología como en la FIGURA 1. Los valores de temperatura de las fuentes termales de Aguas Hediondas son valores máximos reportados recientemente por Carrera y Guevara (2016), aunque para la misma fuente, otros autores anteriores reportan $\mathrm{T}=55^{\circ} \mathrm{C}$ (Beate, 1991) y $\mathrm{T}=46^{\circ} \mathrm{C}$ (Lesmes et al., 2004). 
Una implicación de la variabilidad litológica superficial son las diferencias en calidad de las rocas, que a su vez influyen en las características de la red permeable a niveles someros. En principiolas lavas frescas y masivas tendrían menos permeabilidad que los depósitos no consolidados, pero la presencia de fallas, fracturas y diaclasas hace que incluso las lavas adquieran permeabilidades importantes. Esto es particularmente relevante en lavas que exhiben disyunción columnar por contracción durante enfriamiento, ya que de acuerdo con resultados experimentales se estima que la geometría y dimensiones las columnas influencian significativamente la permeabilidad, pudiendo esta incrementarse varios órdenes de magnitud, con valores máximos medidos cercanos a $10^{-10} \mathrm{~m}^{2}$ (Lamur et al., 2018). En ese sentido, el análisis de estructuras para caracterizar algunas propiedades geotécnicas de los macizos rocosos muestra, de manera semicuantitativa, que la calidad de las rocas, evaluada a partir del $R Q D$ (Bieniawski, 1989) exhibe valor medio $R Q D_{\text {mediana }}=92 \%$ y que la clasificación $R M R$ se sitúa en valor medio $R M R_{\text {mediana }}=69$ (TABLAS 6 y 7). Evidentemente, los valores de resistencia compresiva y tensional dependientes del valor $R M R$, y determinados en este trabajo para cinco afloramientos del CVCCN, son inferiores a los medidos en laboratorio para rocas intactas (Brown, 1981), en concordancia con la observación general de que las resistencias de los edificios volcánicos son mucho menores que las resistencias de los materiales mismos que los conforman (Jerram y Petford, 2011). Las propiedades mecánicas de cinco sitios adicionales en el sector norte del CVCCN fueron también calculadas siguiendo la misma metodología (Bocanegra y Sánchez, 2017), encontrándose los valores medios de $R Q D_{\text {mediana }}=92 \%$ y $R M R_{\text {mediana }}=82$, estos últimos más altos que los calculados en este trabajo. En términos generales, la clasificación $R M R$ y los valores de calidad y resistencia de los macizos rocosos en el CVCCN están en el rango esperado para la mayoría de macizos rocosos (Jerram y Petford, 2011). Así, los estimados geotécnicos y la evidencia morfológica de colapsos en ambos edificios (VC y VCN) (Cortés y Calvache, 1996), sugieren estructuras débiles. De otra parte, en varios afloramientos de lavas se observó dominio de disyunción columnar por enfriamiento (TABLA 3, FIGURA 4), rasgo que aumentaría la permeabilidad de las rocas en superficie (Lamur et al., 2018). Las diferentes litologías, la diversidad de estructuras y discontinuidades, y las propiedades geotécnicas de los macizos también influyen en las condiciones de porosidad y permeabilidad, que en general se observaron variables, tanto a nivel de afloramiento como a nivel regional (TABLAS 3 y 6). Las condiciones de humedad de los afloramientos, y la observación de manantiales o agua de escorrentía en tres sitios reportados aquí (TABLA 3), al igual que las observaciones de Bocanegra y Sánchez (2017) para el sector norte del CVCCN, implican que se favorece la circulación de agua y otros fluidos en niveles superficiales, y se sugiere, con base en las observaciones de campo en rocas y depósitos, que la porosidad y la permeabilidad son moderadas a altas, lo que favorece la circulación de fluidos acuosos entre las áreas de recarga y descarga previamente sugeridas por otros autores (Beate, 1991; INER, 2013) (FIGURAS 1 y 8 ).

Las fuentes de información consultadas reportan que, en el área de interés, las estructuras geológicas mayores (fallas y lineamientos) presentan orientaciones dominantes SW-NE (sistema longitudinal) y SENW (sistema transversal) (Perdomo et al., 1986; Bocanegra y Sánchez, 2017). A nivel de detalle en el presente estudio se reporta que en cuatro sitios se midieron orientaciones de familias de diaclasas en dirección NE y dos sitios mostraban familias de diaclasas en dirección NW (FIGURA 9) ambos grupos de orientaciones consistentes con las tendencias regionales.

El sistema hidrogeológico del altiplano nariñense fue estudiado por INGEOMINAS (2009b), incluyendo marginalmente el área de los volcanes VC y VCN en el extremo SW del altiplano, y cartografiando datos de direcciones de flujo para el agua subterránea en sentido NW-SE y N-S en el área del VC. Aunque en dicha investigación las lavas fueron caracterizadas con porosidad secundaria y sin aparente interés hidrogeológico, el presente estudio aporta nuevos datos que refinan el conocimiento acerca del comportamiento de las aguas superficiales y subterráneas. Desde la actual perspectiva y con base en las observaciones aquí reportadas es razonable sugerir que el agua y los fluidos pueden estar controlados en cierto grado por las estructuras regionales y locales documentadas. 


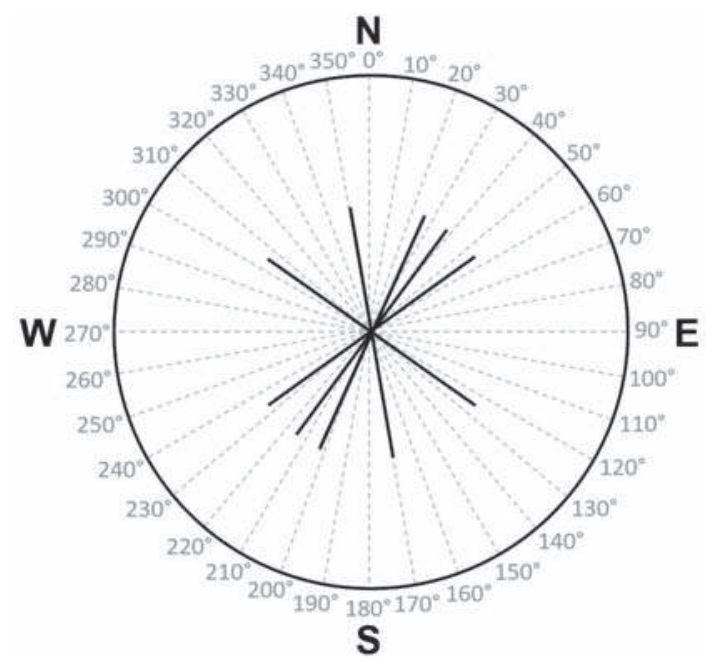

FIGURA 9. Diagrama rosa. Orientación de las estructuras medidas en seis afloramientos, se observa tendencia predominante SW-NE.

El modelo conceptual geotérmico en construcción para el área de interés, de acuerdo a los estudios disponibles, incluye los siguientes elementos (Beate, 1991; Prieto, 2006; CEPAL, 2000; INER, 2013): 1) indicación del área de recarga potencial y dirección de flujo del agua subterránea, 2) dirección de flujo de fluidos calientes en profundidad, 3) configuración general de las isotermas, 4) potencial extensión del reservorio, 5) potencial configuración y extensión de dos fallas, 6) extensión y configuración de una posible capa sello y 7) ubicación de dos sitios de descarga de aguas termales. El presente trabajo aporta los siguientes elementos a dicho modelo conceptual geotérmico: a) sugerencia de posibles complejidades al régimen de recarga y circulación de agua subterránea (flanco oriental del $\mathrm{VCN}$ ); b) tres reportes de posibles áreas adicionales de descarga de aguas termales, una en el costado oriental del VCN (manantial E-019) y dos en el costado oriental del VC (manantial E-008 y laguna E-002); c) la influencia de la geología superficial y el estado geomecánico de las rocas aflorantes, incluyendo la geometría de las diaclasas, la porosidad y la permeabilidad de las rocas en superficie. Por tanto, el presente estudio contribuye al refinamiento de un modelo conceptual preliminar (FIGURA 8), que no incluye lo relacionado a la extensión y configuración de la capa sello del sistema, debido a que este aspecto está fuera del enfoque del presente trabajo.

Finalmente se plantean algunos interrogantes que pueden ser resueltos en estudios posteriores: ¿Cuál es la profundidad más probable de la fuente de calor?, ¿Cuáles son las características fisicoquímicas de las potenciales nuevas áreas de descarga?, ¿Están asociadas las nuevas zonas de descarga reportadas al sistema geotérmico?, ¿Son consistentes las características geológicas y geomecánicas de los afloramientos visitados con sitios ubicados en otros sectores de los volcanes? Estas preguntas pueden resolverse con la integración de resultados de estudios futuros más detallados, los cuales requerirán una aproximación multidisciplinaria.

\section{CONCLUSIONES}

El presente estudio, permite proponer un modelo conceptual geotérmico preliminar para el área del CVCCN, principalmente basado en la valoración de las condiciones generales de aguas y las propiedades geotécnicas de varios macizos rocosos, ubicados en el flanco sur del complejo. Varios sitios, como E8, E10, E12, E18 y E20 fueron clasificados como de "roca húmeda" y "roca empapada", mientras que en los sitios E8 y E19 se encontraron manantiales fríos. Lo anterior implica porosidad y permeabilidad de moderada a alta. Los rangos de valores para algunas propiedades geotécnicas de cinco macizos rocosos en el sector sur del CVCCN son: $90 \leq \% R Q D \leq 96 ; 52 \leq R M R$ $\leq 77 ; 8,89 \leq \sigma_{c m} \leq 35,67 ; \mathrm{y}-1,13 \leq \sigma_{t m} \leq-0,11$. Los valores medios (la mediana como medida de tendencia central) de estos parámetros son: $R Q D_{\text {mediana }}=92 \%$; $R M R_{\text {mediana }}=69 \% ; \quad \sigma_{\text {cm-mediana }}=22,87 \quad \mathrm{MPa} ; \quad$ y $\sigma_{\text {tm- }}$ mediana $=-0,494 \mathrm{MPa}$. Las condiciones superficiales de la litología y las estructuras favorecen la circulación de fluidos en el sistema geotérmico del CVCCN.

\section{AGRADECIMIENTOS}

Los autores expresan su agradecimiento al Departamento de Geociencias, a la Facultad de Ciencias, y a la Universidad Nacional de ColombiaSede Bogotá por el apoyo para la realización de este trabajo. Los comentarios y sugerencias de dos revisores anónimos ayudaron a mejorar la calidad del artículo.

\section{REFERENCIAS}

Alfaro, C. (2015). Improvement of perception of the geothermal energy as a potential source of electrical energy in Colombia, country update. World Geothermal Congress, Melbourne, Australia.

Axelsson, G. (2013). Conceptual models of geothermal systems-introduction. Presented at "Short course $\mathrm{V}$ on conceptual modelling of geothermal systems", El Salvador, February 24-March 2, 
https://orkustofnun.is/gogn/unu-gtp-sc/UNUGTP-SC-16-07.pdf.

Beate, B. (1991). La geotermia: conceptos generales, aplicaciones y estado actual en el ecuador. En: P. Mothes (Ed.). El paisaje volcánico de la sierra ecuatoriana: geomorfología, fenómenos volcánicos y recursos asociados (pp. 71-83). Quito: Corporación Editora Nacional, Colegio de Geógrafos del Ecuador.

Bieniawski, Z.T. (1989). Engineering Rock Mass Classifications: A Complete Manual for Engineers and Geologists in Mining, Civil, and Petroleum Engineering. New York: John Wiley \& Sons.

Bocanegra, L., y Sánchez, J. (2017). Mapa de fallas de los volcanes Chiles - Cerro Negro (Nariño) a partir de minería de datos y confirmación de campo. Boletín de Geología, 39(3), 71-86. doi: 10.18273/revbol.v39n3-2017005.

Brown, E.T. (1981). Rock characterization, testing and monitoring-ISRM suggested methods. Oxford: Pergamon Press.

Carrera, D.V., y Guevara, P.V. (2016). Fuentes termales del Ecuador. Sangolquí: Universidad de las Fuerzas Armadas.

CEPAL. (2000). Proyecto OLADE/CEPAL/GTZ. Estudio para la Evaluación del Entorno del Proyecto Geotérmico Binacional "TufiñoChiles-Cerro Negro". Informe Interno, Comisión Económica para América Latina y el Caribe.

Coe, A.L., Argles, T.W., Rothery, D.A., and Spicer, R.A. (2010). Geological field techniques. Londres: Wiley-Blackwell.

Cortés, G., y Calvache, M. (1996). Investigación sobre la evolución y composición de los volcanes de Colombia: Galeras y Volcanes del Sur. Geología de los Volcanes Chiles y Cerro Negro. Informe Interno. INGEOMINAS, 53p.

Dickson, M., and Fanelli, M. (1995). Geothermal background. In: M. Dickson, M. Fanelli (Eds.). Geothermal energy (pp. 1-36). Chichester: John Wiley \& Sons.

García, Y.K. (2016). Contribuciones geológicas al modelo geotérmico conceptual en la región de los volcanes Chiles-Cerro Negro. Trabajo de Grado, Departamento de Geociencias, Universidad Nacional de Colombia, Bogotá.

Goff, F., and Janik, C.J. (2000). Geothermal systems. In: H. Sigurdsson, B. Houghton, S.R. McNutt, H. Rymer, J. Stix (Eds.). Encyclopedia of volcanoes (pp. 817-834). San Diego: Academic Press.

Gorman, C. (1997). The constructive history and petrology of volcan Cumbal, Southern, Colombia. Master Thesis, Arizona State University, Tempe.

Hoek, E., and Brown, E.T. (1982). Underground Excavations in Rock. London: E \& FN Spon.

Hoek, E., and Brown, E.T. (1997). Practical estimates of rock mass strength. International Journal of Rock Mechanics and Mining Sciences, 34(8), 11651186. doi: 10.1016/S1365-1609(97)80069-X.

INER. (2013). Taller de energía geotérmica-avances en el desarrollo de la geotermia en Ecuador. Instituto Nacional de Eficiencia Energética y Energías Renovables. Consultado el 10 de septiembre de 2015. http://www.iner.gob.ec/.

INGEOMINAS. (1999). Atlas de amenaza volcánica en Colombia. Informe Interno, 121p.

INGEOMINAS. (2009a). Guía metodológica para el reconocimiento geológico en campos geotérmicos. Informe interno, $31 \mathrm{p}$.

INGEOMINAS. (2009b). Evaluación hidrogeológica regional del Altiplano nariñense. Informe interno, $67 \mathrm{p}$.

Irvine, T.N., and Baragar, W.R.A. (1971). A guide to the chemical classification of the common volcanic rocks. Canadian Journal of Earth Sciences, 8(5), 523-548.

ISAGEN S.A ESP (2012). Notas para la investigación y desarrollo de proyectos geotérmicos en Colombia. 78p.

Janoušek, V., Farrow, C.M., and Erban, V. (2006). Interpretation of whole-rock geochemical data in igneous geochemistry: introducing Geochemical Data Toolkit (GCDkit). Journal of Petrology, 47(6), 1255-1259. doi: 10.1093/ petrology/egl013. 
Jerram, D., and Petford, N. (2011). The field description of igneous rocks. Oxford: Wiley-Blackwell.

Lamur, A., Lavallée, Y., Iddon, F.E., Hornby, A.J, Kendrick, J.E., von Aulock, F.W., and Wadsworth, F.B. (2018). Disclosing the temperature of columnar jointing in lavas. Nature Communications, 9, 1-7. doi: 10.1038/s41467-018-03842-4.

Le Bas, M., Le Maitre, R., Streckeisen, A., and Zanettin, B. (1986). A chemical classification of volcanic rocks based on the total Alkali-Silica diagram. Journal of Petrology, 27(3), 745-750. doi: 10.1093/ petrology/27.3.745.

Lesmes, L., Bobadilla, L., Hernández, M., Cañón, Y., Mojica, J. and Garzón, G. (2004). Mineralogía $\mathrm{y}$ fisicoquímica de las fuentes termales del Departamento de Nariño. Boletín de Geología, 26(42), 57-66.

Mejía, E., Rayo, L., Méndez, J., and Echeverri, J. (2014). Geothermal development in Colombia. Short course VI on Utilization of Low-and MediumEnthalpy Geothermal Resources and Financial Aspects of Utilization. El Salvador.

Miyashiro, A. (1974). Volcanic rocks series in island arcs and active continental margins. American Journal of Science, 274(4), 321-355. doi: 10.2475/ ajs.274.4.321.

Moeck, I.S. (2014). Catalog of geothermal play types based on geologic controls. Renewable and Sustainable Energy Reviews, 37, 867-882. doi: 10.1016/j.rser.2014.05.032.

Nelson, W. (1962). Contribución al conocimiento de la Cordillera Occidental. Sección carretera Cali - Buenaventura. Boletín Geológico, 10(1-3), 81108.
Peccerillo, A., and Taylor, S.R. (1976). Geochemistry of eocene calc-alkaline volcanic rocks from the Kastamonu area, northern Turkey. Contributions to Mineralogy and Petrology, 58(1), 63-81. doi: 10.1007/BF00384745.

Perdomo, G., Ardila, R., y Meneses, L. (1986). Estudio geológico para prospección de azufre en el área de Cumbal - Chiles - Mayasquer (Nariño). Tesis de grado, Universidad Nacional de Colombia, Bogotá, Colombia.

Pinilla, A., Ríos, P., Rodríguez, B., Sánchez, J., Pulgarin, B., Borrero, C., y Roa, H. (2008). El Neógeno Volcánico en el Altiplano Nariñense, suroccidente colombiano. Geología Colombiana, 33, 69-78.

Prieto, A. M. (2006). Diagnóstico del potencial geotérmico en la zona del Proyecto Geotérmico Binacional Tufiño Chiles Cerro Negro. Trabajo de grado, Departamento de Geociencias, Universidad Nacional de Colombia, Bogotá, Colombia.

Streckeisen, A. (1973). Plutonic Rocks: Classification and Nomenclature Recommended by the IUGS. Sub-Commission on the Systematic of Igneous Rocks. Geotimes, 18, 26-30.

Velásquez, M.E., y Parra, E. (2002). Geología de la plancha 447 - Ipiales y 447BIS - Tallambí. Memoria Explicativa. INGEOMINAS, 118.

\begin{tabular}{c}
\hline \hline Yohanna K. García \\
ORCID: 0000-0002-7188-8911 \\
John J. Sánchez \\
ORCID: 0000-0002-9024-7554 \\
\hline \hline
\end{tabular}

Trabajo recibido: enero 26 de 2017

Trabajo aceptado: septiembre 25 de 2018 